\title{
A computational model for analysing interactive buckling and delamination growth in composite structures
}

\author{
B P NAGANARAYANA ${ }^{1}$ and $S$ N ATLURI ${ }^{2}$ \\ ${ }^{1}$ Structures Division, National Aerospace Laboratories, Bangalore 560017, \\ India \\ ${ }^{2}$ Georgia Institute of Technology, Atlanta, GA 3033-0356, USA \\ e-mail: naga@cmmacs.ernet.in; naga@csnal.ren.nic.in; satya.atluri@cad. \\ gatech.edu
}

\begin{abstract}
In this paper, a unified method is presented: (i) to model delaminated stiffened laminated composite shells; (ii) for synthesising accurate multiple post-buckling solution paths under compressive loading; and (iii) for predicting delamination growth. A multi-domain modelling technique is used for modelling the delaminated stiffened shell structures. Error-free geometrically nonlinear element formulations - a 2-noded curved stiffener element (BEAM2) and a 3-noded shell element (SHELL3) - are used for the finite element analysis. An accurate and simple automated solution strategy based on Newton type iterations is used for predicting the general geometrically nonlinear and postbuckling behaviour of structures. A simple method derived from the 3-dimensional $J$-integral is used for computing the pointwise energy release rate at the delamination front in the plate/shell models. Finally, the influence of post-buckling structural behaviour and the delamination growth on each other has been demonstrated.
\end{abstract}

Keywords. Multi-domain modelling; quasi-conforming elements; delamination growth; $J$-integral; automated post-buckling solution.

\section{Introduction}

The laminated and stiffened structures are particularly prone to interlaminar debonding (delamination) type of failures since the interlaminar bond strength is much less when compared to in-plane laminar strength. Such delaminations can be caused, at any time, under several design and operating conditions e.g. large transverse stresses, tapering of the laminate, clamping in a vice, drilling a hole, low velocity impact such as dropping a tool during maintenance etc. (figure 1). In addition, structural fatigue and environmental factors like moisture, temperature and corrosion often weaken the interlaminar bond-strength and hence accelerate formation/propagation of the delaminations. 
(a)

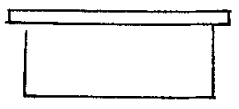

(b)
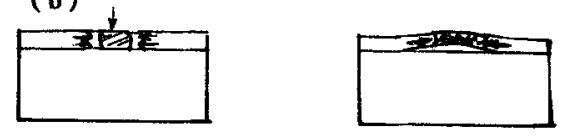

(c)

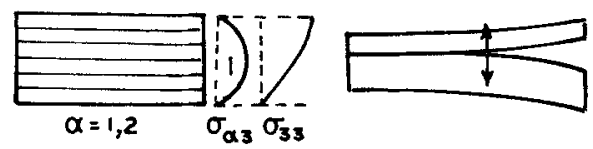

(d)
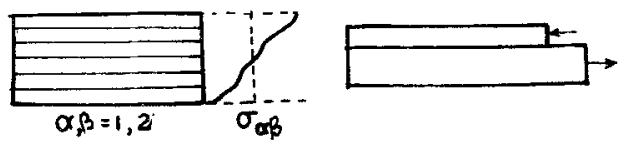

(e)

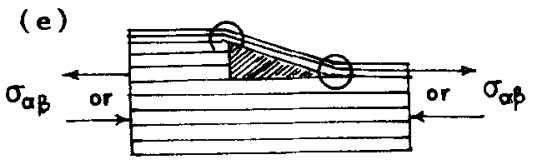

Figure 1. Causes for initiation and growt th of delamination failure - (a) Presstressed plates; (b) impact/indentation loads; (c) transverse shear/normal stresses; (d) in-plane shear/normal stresses; (e) laminate tapering; (f) ply-failure under operating conditions. The respective causes for delamination are as follows. (a) Hygro-thermo-mechanical compressive stresses due to fabrication and/or drawing defects; (b) plastic zone under impact load leading to material failure and local partial layer separation. The resulting compressive layer forces can cause delamination; (c) local bonding material failure due to high transverse stresses often cause delamination, particularly near the free edges, plies debond in the opening mode; (d) high in-plane stresses cause ply-failure, which in turn accelerates the delamination process; (e) high local bending stress concentrations in the encircled zones may force the laminae to separate at the corners; (f) as in (d), ply failure under any operating conditions accelerates the delamination process.

The delaminations are particularly dangerous because: they generally reduce the overall laminate strength due to material discontinuity; they act as imperfections when located eccentrically, and thus substantially reduce the overall buckling strength of the laminate; and they grow rapidly under in-plane compressive loads - since the delamination often buckles locally much earlier than global structural buckling - resulting in a progressive reduction in laminate strength and increase in delamination growth rate, finally leading to fatal failure. Also, stiffened delaminated structures can buckle in multiple levels - local delaminate, laminate, panel, stiffener and structural - often accelerating the delamination growth dramatically.

In addition, delaminations are very often hidden and escape simple inspection and have very high potential to grow under operating conditions. Thus an a priori assessment of: the nature and magnitude of delamination that could be induced under specified circumstances; the growth rate of the delamination under specified loading environments and structural instability; the reduction in laminate strength due to the presence and the growth of a delamination; and possible methods for avoiding the damage 
and/or arresting/controlling the damage growth, become essential so that the designer can build these considerations into his basic design. Accordingly, these problems have been addressed widely over the last two decades from both experimental and theoretical points of view. In this paper we shall limit ourselves to a reliable computation of the post-buckling structural behaviour and the pointwise energy release rate distribution along the delamination front in a laminated composite structure. The energy release rate computed here can be used as an effective feedback to the designer to check whether an existing delamination is a potential danger from the structural integrity point of view.

Though the delaminations are prone to grow under a variety of loading configurations, it is understood that they are extremely sensitive to the buckling loads. Under such loads, they can reduce the overall buckling strength of the laminate considerably and can also grow dramatically under postbuckling loads, leading to structural failure. Very often, delaminated composites can be modelled as problems of plate-bending, using any of the theories of plates and shells that are well-established. Furthermore, laminate deformation is mostly elastic and hence the different energetic measures established in linear elastic fracture mechanics are meaningful for characterising delamination growth. Use of these energetic measures in conjunction with an analysis of the post-buckling behaviour of the delaminated plates often results in a simple a posteriori expression for the pointwise energy release rate distribution along the delamination front as demonstrated in this paper. Thus, modelling and analysis of coupled failure mechanisms in stiffened delaminated structures - which were once formidable - are being reconsidered with renewed confidence in recent years.

Most of the researchers in the past have concentrated on the very thin near-surface rectilinear and/or circular shaped delaminations in a homogeneous isotropic material medium (Kachanov 1976; Chai et al 1981; Bottega \& Maewal 1983; Evans \& Hutchinson 1984; Yin 1984, 1985). This is because, under certain assumptions (known as 'thin film' assumptions), the deformations of such delaminations can be studied, in isolation, as problems of clamped plates under compressive loads and one can obtain quasi-analytical estimates of delamination growth under simple loading conditions e.g. biaxial load. Recently, attempts were made to obtain solutions for elliptic delaminations e.g. using the Rayleigh-Ritz technique and certain geometric constraints to couple the post-buckling large deffections and membrane deformation (Flanagan 1988). Generally, such solutions are valid for very thin delaminations in thick laminates. But, most of the practical laminate panels, for example in aerospace applications, are generally thin and very often the delaminate thickness is comparable to the total laminate thickness or to the base thickness (see figure 2). Also, such solutions (e.g. Evans \& Hutchinson 1984) are generally based on assumptions of quasi-linear local post-buckling behaviour of the delaminate plate and hence are valid only in the vicinity of the range of the load for local buckling of the delaminate. In practice, however, the local delaminate post-buckling behaviour is very nonlinear; and also the laminated panels are often allowed to buckle even globally. Finally, the presently available analytical or quasi-analytical solutions are limited to a single delamination of a standard shape and location only in plates of standard topology and boundary conditions.

Today, one would turn to the finite element method for a general computational analysis of arbitrarily shaped stiffened composite laminates. However, an analysis of the 

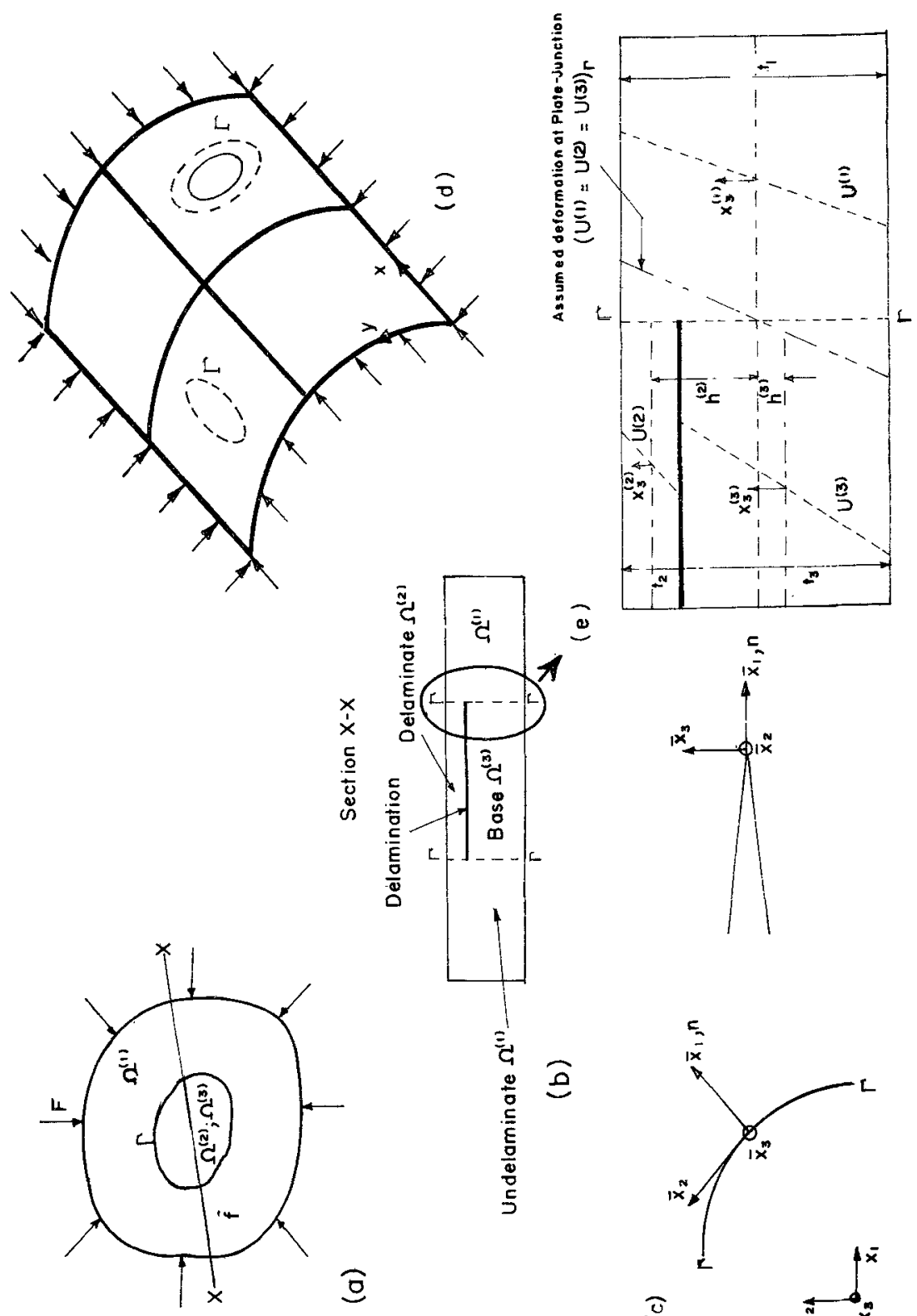

离

菾

章

สี

e

iิ

苛

च

它

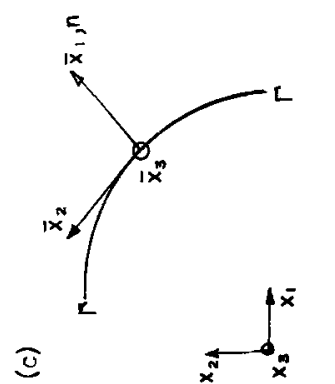

总

ง

을 
growth of embedded delaminations requires 3-dimensional modelling (Whitcomb 1989) associated with a sophisticated geometrically nonlinear post-buckling solution capability. Hence, if a finite element method is used, the analysis becomes extremely expensive from both computer memory and time points of view. Even though one can think of a global 2-dimensional post-buckling analysis and a local 3-dimensional growth analysis using established methods such as alternating technique (Nishioka \& Atluri 1981), virtual crack extension technique (Parks 1974), modified crack-closure technique (Rybicki \& Kanninen 1977), etc. the procedure is more involved and still expensive to use extensively for each incremental solution in a cycle of finite element post-buckling solutions.

In the early $80 \mathrm{~s}$, a cost-effective one-dimensional model - the so-called multi-plate model - was proposed to model a laminate with a single delamination (Chai et al 1981). This procedure, however, can be extended to handle multiple delaminations of different shapes and locations in general composite plates and shells as well (Naganarayana et al 1995). Here, the delaminated structure is modelled as an assembly of three distinct parts, namely, laminate, base and delaminate (figure 2). The well-established theories of plates/shells can be readily used for modelling each of the three plates. Normally, the same plate theory is used to establish the continuity conditions at the joint between them at the delamination front. However, the one-dimensional method presented in Chai et al (1981) for computing the energy release rate uses a simple numerical derivative of the total potential energy and hence requires two computations - one for crack diameter $2 a$ and another for the extended crack diameter $2(a+\mathrm{d} a)$. This makes it cumbersome and expensive to use in practice extensively, especially for 2-dimensional planar delaminations of arbitrary shapes and locations. Today, reliable energy-based parameters (such as the $J$-integral, the equivalent domain integral etc.) and computational techniques (such as the alternating methods, virtual crack extension, modified crack closure etc.) are established in the field of linear elastic fracture mechanics for predicting the crack growth in a much simpler fashion. Recently, some of these techniques were extended for characterising delamination growth in a multi-plate model, e.g. virtual crack extension method (Gilletta 1988), modified crack closure technique (Whitcomb \& Shivakumar 1989), VCCTS (virtual crack closure technique step-by-step) approach (Tsao et al 1991).

In this paper, we consider the following 3-dimensional energy-based parameters to derive simple expressions for the pointwise energy release rate at any point on the front of an arbitrary-shaped planar delaminations in composite laminates: the $J$-integral (Rice 1968) computed along a closed surface of an infinitesimal radius enclosing the crack tip; and the equivalent domain integral (Nikishkov \& Atluri 1987) computed over a finite annular volume with the inner surface of an infinitesimal radius enclosing the crack tip. These parameters are suitably modified for the present problem of plate/shell flexure; and using assumptions that characterise the delamination growth and plate/shell flexure, simple expressions are derived for the pointwise energy release rate distribution along the delamination front (Naganarayana \& Atluri 1995a,b). The techniques presented here, for delamination growth prediction, can be used in an a posteriori sense in conjunction with any analytical/computational method of post-buckling analysis of plates that can take care of appropriate multi-point constraints at the delamination front. However, in this paper, an 
in-house finite element software-NONCAT: NONlinear Computational Analysis Tool for structural analysis (Huang et al 1995) - incorporating curved stiffener and shell elements, an automated nonlinear post-buckling solution, and multi-domain modelling technique, is used for the analysis.

Here, the multi-domain modelling technique (Naganarayana \& Huang 1995) is used to model the delaminated plates/shells. A 3-noded triangular quasi-conforming curved shell element (SHELL3) is used for modelling the delaminated sublaminates and the nondelaminated plate/shell. A 2-noded curved beam element (BEAM2) is used for modelling the stiffeners. The stiffener element (Naganarayana \& Prathap 1996) is developed based on the Euler-Bernoulli theory of beam flexure in a curvilinear coordinate system. The shell element (Huang et al 1994) is based on a classical shallow shell theory, again, described in a curvilinear coordinate system. The causes for membrane locking and nonlinear locking are identified and eliminated from the element formulations, using reduced integration for the membrane strain energy (Naganarayana et al 1995). The transverse shear strain energy is included into the formulation explicitly in accordance with the Reissner-Mindlin theory of plate flexure with the transverse shear strains as nodal degrees of freedom. Therefore the elements do not sense shear locking either. In case of the shell element, the $C^{0}$-continuity is exactly preserved for the field variables. However, the $C^{1}$-continuity required for the transverse deflection across the element boundaries is achieved a posteriori in a weak form - i.e. in a quasi-conforming sense (Huang et al 1994).

An automated incremental general nonlinear and post-buckling Newton type solution strategy, incorporating an arc-length controlled load incrementation, and branch switching based on a linearised asymptotic solution (Huang \& Atluri 1995), is utilised while using the displacement type finite element model. The stresses are post-processed for each load increment, to obtain pointwise energy release rate distribution along the delamination front, by using the adapted $J$-integral and equivalent domain integral approaches (Naganarayana \& Atluri 1995a,b) mentioned above.

In this paper, we present the complete computational strategy for structural and finite element modelling of delaminated and/or stiffened laminated plates/shells; automated geometrically nonlinear and post-buckling solution strategy for the finite element model; and delamination growth assessment in terms of the pointwise energy release rate distribution along the delamination front. Different aspects briefly discussed, are related to structural modelling, finite element formulations and possible errors involved, solution strategies that can pass the instability points and switch the solution branches if necessary, energy release rate prediction and interaction between post-buckling structural deformation and delamination growth. Also, the structure of the software NONCATS that involves these strategies is briefly explained. Finally, a few examples are presented to demonstrate how the present computational model functions.

\section{Structural modelling}

Here, for the sake of convenience and simplicity of presentation, we shall consider a laminated composite shell with a single delamination of an arbitrary shape and location, 
subjected to arbitrary compressive loads (figure 2). The structure is modelled using the multi-domain model (Naganarayana \& Huang 1995) wherein the delaminated shell is assumed to be assembled with three distinct shells: (1) Laminate: nondelaminated zone $\Omega^{(1)}$; (2) Delaminate: thinner side of the delaminated zone $\Omega^{(2)}$; and (3) Base: thicker side of the delaminated zone $\Omega^{(3)}$. The three shells, $\Omega^{(i)}, i=1,2,3$ respectively, have midsurface areas $\mathcal{A}^{(i)}$; thicknesses $t^{(i)}$; boundaries $\partial \Omega^{(i)}$; and midsurface boundaries $\partial \mathcal{A}^{(i)}$. The delamination edge is denoted by $\Gamma$. The assumptions of the Reissner-Mindlin theory of plate bending are used for modelling each shell and the joint between them. Thus, for each shell, the 3-dimensional displacement field ( $\left.\mathbf{U} \equiv\left\{\begin{array}{lll}U_{1} & U_{2} & U_{3}\end{array}\right\}\right)$ can be expressed in terms of the corresponding midsurface displacement $\left(\mathbf{u} \equiv\left\{\begin{array}{lll}u_{1} & u_{2} & u_{3}\end{array}\right\}\right)$ and rotation $\left(\boldsymbol{\theta} \equiv\left\{\theta_{1} \theta_{2} 0\right\}\right)$ fields as,

$$
\mathbf{U}^{(i)}\left(x_{\alpha}, x_{3}\right)=\mathbf{u}^{(i)}\left(x_{\alpha}\right)-x_{3}^{(i)} \theta^{(i)}\left(x_{\alpha}\right),
$$

where $x_{\alpha}^{(i)}(\alpha=1,2)$ are the in-plane curvilinear shell coordinates and $x_{3}^{(i)}$ is the thickness coordinate for the $i$ th $(i=1,2,3)$ shell (figure 2 ). The structural continuity at the delamination front $\Gamma$ is maintained by assuming the deformation to be unique at the junction of the three shells i.e. $\mathbf{U}^{(1)}=\mathbf{U}^{(2)}=\mathbf{U}^{(3)}$ on $\Gamma$. In other words, at the delamination edge, the mid-surface degrees of freedom of the delaminate and the base shells are assumed to be related to those of the nondelaminated shell by,

$$
\left(\begin{array}{l}
u_{3}^{(1)}=u_{3}^{(2)}=u_{3}^{(3)} \\
\theta_{\alpha}^{(1)}=\theta_{\alpha}^{(2)}=\theta_{\alpha}^{(3)} \\
u_{\alpha}^{(i)}=u_{\alpha}^{(1)}+h^{(i)} \theta_{\alpha}^{(1)}
\end{array}\right)_{a t \Gamma}
$$

where $h^{(i)}$ is the distance of the midsurface of the $i$ th shell from the laminate midsurface (figure 2). It can be noted that the above continuity conditions at the delamination edge can be modified appropriately when using any other alternative plate/shell theory (e.g. higher order shear deformable theory) or by choosing appropriate heuristic multi-point constraints based on experience.

Similarly, the beam (stiffener) degrees of freedom are related to the shell degrees of freedom such that the transverse variations of deformation across the shell and beam section are consistent with the Reissner-Mindlin theory:

$$
\begin{aligned}
u_{3}^{b} & =u_{3}^{s}, \\
\theta_{\alpha}^{b} & =\theta_{\alpha}^{s}, \\
u_{\alpha}^{b} & =u_{\alpha}^{s}+e \theta_{\alpha}^{s},
\end{aligned}
$$

where superscripts $b$ and $s$ represent beam and shell degrees of freedom respectively, and $e$ is the eccentricity of the stiffener's neutral axis with reference to the neutral surface of the shell.

In this paper, a 2-noded curved beam element (BEAM2) (figure 3) and a 3-noded curved shell element (SHELL3) (figure4) are used for modelling stiffened structures. The elements 


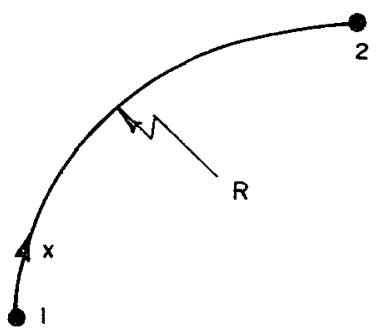

Figure 3. BEAM2: 2-noded curved stiffener/beam element.

are described in a curvilinear coordinate system and are based on a quasi-conforming formulation (Huang et al 1994).

In the current formulation, a classical $C^{1}$-continuous field description is used and the transverse shear strain components are exclusively introduced as generalised degrees of freedom conforming with the Reissner-Mindlin theory:

$$
\gamma_{\alpha 3}=\left(w,_{\alpha}+b_{\alpha \beta} u_{\beta}\right)-\theta_{\alpha}=\phi_{\alpha}-\theta_{\alpha},
$$

where $b_{\alpha \beta}$ is the curvature tensor of the shell's mid-surface. Substituting (1) and (4) in the regular 3-dimensional strain tensor, we get the membrane, the flexural and the transverse shear strain components respectively as:

$$
\begin{aligned}
& \epsilon_{\alpha \beta}=\frac{1}{2}\left(u_{\alpha, \beta}+u_{\beta, \alpha}\right)+\frac{1}{2} \phi_{\alpha} \phi_{\beta}-b_{\alpha \beta} w, \\
& \chi_{\alpha \beta}=\frac{1}{2}\left(\gamma_{\alpha 3, \beta}+\gamma_{\beta 3, \alpha}\right)-\frac{1}{2}\left(\phi_{\alpha, \beta}+\beta \beta, \alpha\right), \\
& \epsilon_{\alpha 3}=\frac{1}{2} \gamma_{\alpha 3} .
\end{aligned}
$$

The finite element formulations, hence, use the seven degrees of freedom $-u_{1}, u_{2}, w$, $w_{1}, w_{2}, \gamma_{13}, \gamma_{23}$ - to define the strain components and hence the structural deformation. It can be observed here that the curved shell element (SHELL3) needs $C^{0}$-continuous interpolation functions (e.g. Lagrangian) for the inplane displacement components $\left(u_{1}\right.$, $\left.u_{2}\right)$ and the transverse shear strain components $\left(\gamma_{13}, \gamma_{23}\right)$ and $C^{1}$-continuous interpolation functions (e.g. Hermetian) for the transverse deflection $(w)$. A compatible field-description is used in the curved beam formulation as well.

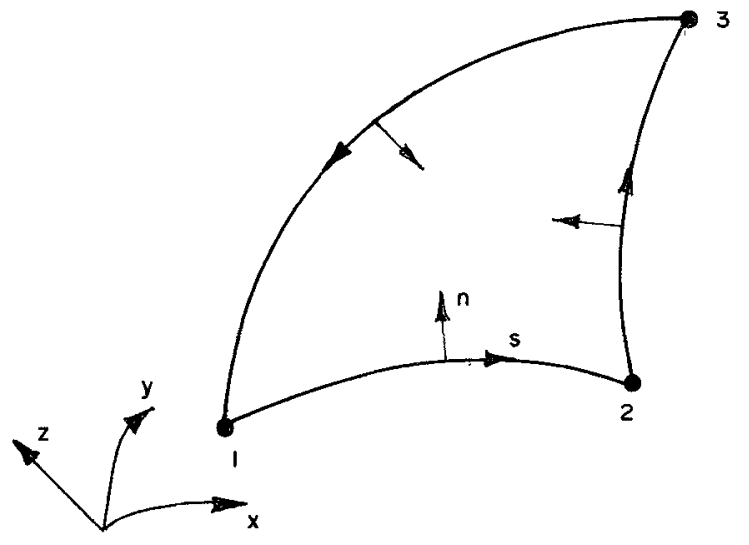

Figure 4. SHELL3: 3-noded curved shell element. 
It is interesting to note here that the transverse shear strains vanish in a variationally correct sense as the structural thickness decreases and the effect of the transverse shear deformation vanishes from the flexural strains in a consistent manner. Thus, such an element formulation is free of shear locking.

However, these elements suffer from membrane locking when used to model curved structures, particularly in the regime of inextensional bending (Babu \& Prathap 1988). This is due to the inconsistent participation of the terms $b_{\alpha \beta} w$ with reference to the basic membrane strains $\frac{1}{2}\left(u_{\alpha, \beta}+u_{\beta, \alpha}\right)$. Similarly, inconsistent participation of the nonlinear terms $\frac{1}{2} \phi_{\alpha} \phi_{\beta}$ with reference to the basic membrane strain components lead to the so-called nonlinear locking (Naganarayana \& Prathap 1996) when used to model geometrically nonlinear systems in the limits of inextensional bending. In the curved beam and shell elements considered here, the membrane strain energy is computed using a reduced order of Gaussian quadrature so that both the locking phenomena are eliminated from these elements (Naganarayana et al 1995) based on the understanding gained from a one-dimensional beam element formulation (Naganarayana \& Prathap 1996).

The shell element is required to satisfy $C^{1}$-continuity requirements (for the transverse deflections) over the element domain as well as across the element boundary. $C^{1}$-continuous shape functions (e.g. Hermitian) are used to interpolate the transverse deflection over the element domain. The $C^{1}$-continuity requirements across the element boundary are however satisfied a posteriori in a weak form - quasi-conforming field-description - using the $\mathrm{Hu}-$ Washizu variational principle (Huang et al 1994).

As mentioned in the previous section, the sublaminate degrees of freedom (at the delamination front) and the stiffener degrees of freedom are related to the corresponding laminate degrees of freedom using the multi-point-constraints in accordance with the Reissner-Mindlin theory of plate flexure. One may refer to Naganarayana et al (1995), Naganarayana \& Prathap (1996) and Huang et al (1994) for detailed description of the element formulations and finite element modelling/analysis of the delaminated stiffened composite structures.

\section{Delamination growth prediction}

Delamination is a typical form of failure in laminated structures occurring purely due to failure of the interlaminar bond. Normally, in laminated composite structures, interlaminar bond strength is much less when compared to laminar strength. Thus, unlike other forms of failure (e.g. inter-laminar cracks, spalling etc.) which may start and grow under severe loads and/or fatigue, delaminations may take place at much lower loads and could grow very rapidly even under normal maintenance and operating conditions (figure 1) leading to structural failure. In addition, they very often escape visual inspection. Therefore, extra care has to be taken for containing the delamination formation and its growth.

In this section, a computational model is derived to predict delamination growth in terms of pointwise energy release rate. It is assumed that delaminations start and grow in the interlaminar bond region. Therefore, the delamination and its growth take place in a 
homogeneous medium so that the growth can be assumed to be self-similar. Therefore, the $J$-integral (or the equivalent domain integral) representing only self-similar crack growth is meaningful in the present case. Here, the 3-dimensional $J$-integral and the equivalent domain integral are used to compute the strain energy release rates.

The pointwise energy release rate for 3-dimensional self-similar crack growth, $(\mathcal{G}(\Gamma)$ ), is defined as (Atluri 1986)

$$
\begin{aligned}
\mathcal{G}(\Gamma) \Delta \Gamma= & \lim _{e \rightarrow 0} \int_{A_{e}}\left[W \bar{n}_{1}-\bar{\sigma}_{\alpha \beta} \bar{n}_{\beta} \frac{\partial \bar{U}_{\alpha}}{\partial \bar{x}_{1}}\right] \mathrm{d} A \\
& +\int_{A_{1}} \bar{\sigma}_{\alpha 2} \frac{\partial \bar{U}_{\alpha}}{\partial \bar{x}_{1}} \mathrm{~d} A-\int_{A_{2}} \bar{\sigma}_{\alpha 2} \frac{\partial \bar{U}_{\alpha}}{\partial \bar{x}_{1}} \mathrm{~d} A,
\end{aligned}
$$

where, $\alpha, \beta=1,2,3 ; A_{e}$ is the area of the tube of radius $e$ enclosing the crack front; $A_{1}$ and $A_{2}$ are the areas covering the ends of the tube (figure 5); and $\bar{\sigma}, \bar{U}$ and $\bar{n}$ are defined in the crack tip coordinate system $\overline{\mathbf{x}}$ (figure 2 ).

For self-similar crack growth in homogeneous media, the path-independence of the $J$ integral is maintained (Naganarayana \& Atluri 1995a,b) and hence the infinitesimal tube enclosing the crack edge can have a cross-section of any shape. Considering a rectangular tube enclosing the delamination front and passing through the nearest stress recovery points $\left(S^{(i)}\right)$ of the adjoining elements (figure 5); applying the assumptions of the theory of plate flexure that is used to model the laminate and the delaminated sublaminates; and carrying out the integration through the thickness for each sublaminate, we get the pointwise energy release rate as a simple function of the stress resultants, the displacement gradients and the strain energy densities at the points $\left(S^{(i)}\right)$ as,

$$
\mathcal{G}_{g}(\Gamma)=\mathcal{F}_{g}\left[\hat{W}-\left(\bar{N}_{1 \alpha} \bar{u}_{\alpha, 1}+\bar{M}_{1 \alpha} \bar{\theta}_{\alpha, 1}+\bar{Q}_{13} \bar{u}_{3,1}\right)\right]
$$

where $\mathcal{F}_{g}(*)=(*)_{g^{(1)}}-(*)_{g^{(2)}}-(*)_{g^{(3)}}$ and $(*)_{g^{(i)}}$ corresponds to the quantities $(*)$ evaluated at specified points (generally Gauss points) on the annular surface.

It is interesting to observe here that, if the rectangular tube is shrunk to the surface along the thickness coordinate at the delamination edge, we get the pointwise energy release rate in terms the nodal values as,

$$
\mathcal{G}_{n}(\Gamma)=\mathcal{F}_{n}\left[\hat{W}-\left(\bar{N}_{1 \alpha} \bar{u}_{\alpha, 1}+\bar{M}_{1 \alpha} \bar{\theta}_{\alpha, 1}+\bar{Q}_{13} \bar{u}_{3,1}\right)\right]
$$

where $\mathcal{F}_{n}(*)=(*)_{n}(1)-(*)_{n}(2)-(*)_{n}(3)$ and $(*)_{n}(i)$ corresponds to the quantities $(*)$ evaluated at specified nodes.

However, it is a well-known fact that the stresses, displacement gradients and strain energy density are more accurate at the optimal stress recovery points (Barlow 1976; Prathap 1993) than at the nodes in finite element analysis. Therefore, $\mathcal{G}_{g}(\Gamma)(7)$ with the $J$-enclosure passing through the Barlow points is always the most reliable estimate.

The local stress resultants $(\overline{\mathbf{N}}, \overline{\mathbf{M}}, \overline{\mathbf{Q}})$ and displacement gradients $\left(\bar{U}_{\alpha, \beta}\right)$ can be obtained from their global Cartesian counterparts $\left(\mathbf{N}, \mathbf{M}, \mathbf{Q} ; U_{\alpha, \beta}\right)$, by applying the regular tensorial transformations between the reference coordinate system $\mathbf{x}$ and the crack tip coordinate system $\overline{\mathbf{x}}$. 
(a)
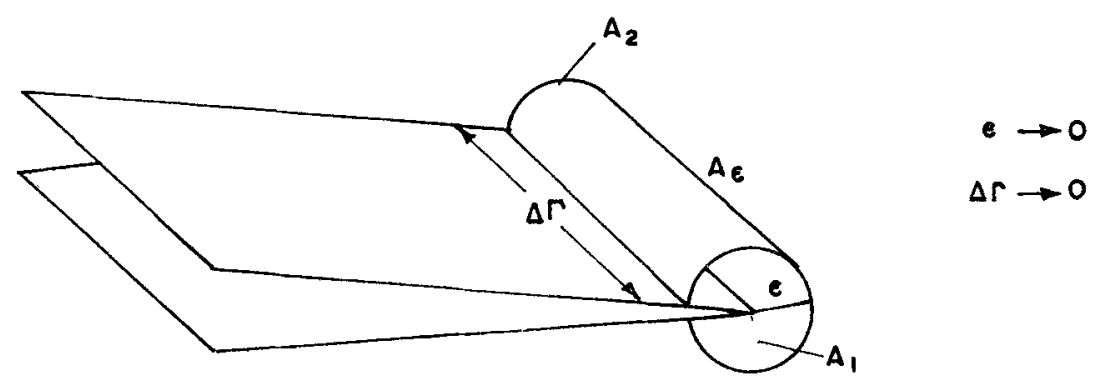

\section{(b)}

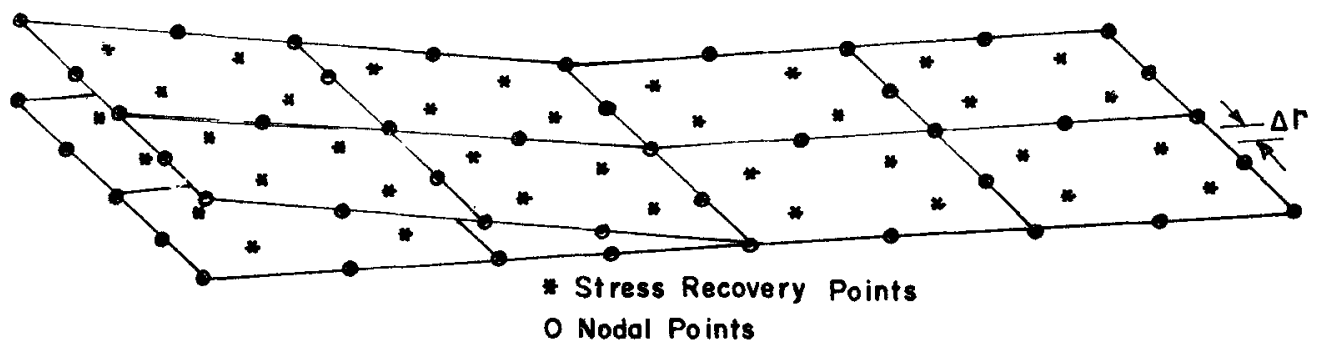

(c)

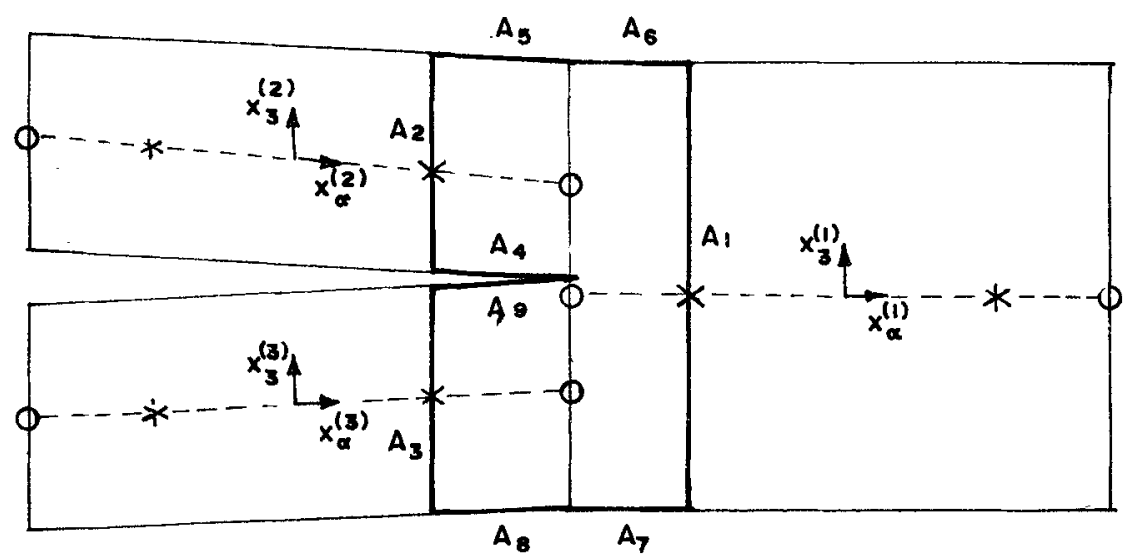

Figure 5. $J$-integral for self-similar delamination growth in a plate/shell model: (a) The $J$-enclosure; (b) the finite element model; (c) the modified $J$-enclosure for the shell model.

Recently, the 3-dimensional equivalent domain integral (EDI) (Nikishkov \& Atluri 1987) interpretation for the $J$-integral was modified to the present problem by choosing appropriate enclosures around the crack tip and the so-called $s$-functions in accordance with the Reissner-Mindlin theory of flexure (Naganarayana \& Atluri 1995a). Then, we get the pointwise energy release rate as a weighted average function of the stress resultants, 
the displacement gradients and the strain energy densities in the elements adjacent to the delamination edge, as,

$$
\mathcal{G}_{e}(\Gamma)=\overline{\mathcal{F}}_{e}\left[\hat{W}-\left(\bar{N}_{1 \alpha} \bar{u}_{\alpha, 1}+\bar{M}_{1 \alpha} \bar{\theta}_{\alpha, 1}+\bar{Q}_{13} \bar{u}_{3,1}\right)\right],
$$

where

$$
\overline{\mathcal{F}}_{e}(*)=\frac{1}{\Delta_{1}} \int_{\Delta_{1}}(*) \mathrm{d} \Delta-\frac{1}{\Delta_{2}} \int_{\Delta_{2}}(*) \mathrm{d} \Delta-\frac{1}{\Delta_{3}} \int_{\Delta_{3}}(*) \mathrm{d} \Delta .
$$

Again, by applying the required tensorial transformations on the global stress resultants $(\mathbf{N}, \mathbf{M}, \mathbf{Q})$ and displacement gradients $\left(U_{\alpha, \beta}\right)$, their counterparts in the crack tip coordinate system $\left(\overline{\mathbf{N}}, \overline{\mathbf{M}}, \overline{\mathbf{Q}} ; \bar{U}_{\alpha, \beta}\right)$ can be computed.

Thus the pointwise energy release rate derived from the equivalent domain integral approach may be more meaningful when compared to that derived from the regular $J$ integral approach since the former can capture the variation of different parameters along the normal to the crack front in the vicinity of the crack tip in a better fashion. However, when a constant stress/strain element is used to model the problem, the energy release rate computed in (9) becomes identical to that derived directly from the $J$-integral ((7) and (8)).

The present exercise provides the energy release rate as a design parameter to estimate the critical loading condition for a laminated composite structure with a specified delamination embedded in the structure. The critical energy release rate that an interlaminar bond can withstand may be obtained by an appropriate material database.

\section{Incremental nonlinear and post-buckling solution strategies}

In a delaminated structure, the delamination normally acts as a geometric imperfection such that the structure is susceptible to buckling under compressive loads. Very often, the delaminate configuration is such that the delaminated sublaminate(s) buckles locally much earlier to laminate/structural buckling. The locally buckled sublaminates will often lead to premature global buckling since the original geometric imperfections are now highly accentuated. Thus, the structure may experience multiple post-buckling deformations which are highly coupled with each other and simultaneous occurrence of different types of instabilities - limit or bifurcation points. Also, post-buckling structural performance interacts with the delamination growth as well. Therefore, an automated incremental nonlinear solution strategy becomes very important for tracing the multiple post-buckling deformation modes in a delaminated stiffened composite structure.

In the finite element context, such an exercise normally involves setting the incremental equilibrium equations for the structure, appropriate iteration techniques (e.g. NewtonRaphson) with a regular solver (e.g. Gauss elimination) for obtaining incremental solutions, appropriate initial load increment to avoid divergence and to ensure progressive path tracing, automated identification and classification of singular points (limit points and bifurcation points), automated branch switching to trace the desired post-buckling solution in case of bifurcation problems, monitored equilibrium increments for assured convergence in spite of the presence of singular points, and finally obtaining the required incremental solutions (displacements and stresses). 
Generally, in incremental nonlinear finite element analysis (FEA), new incremental solution is sought at the unknown point $(\mathbf{q}+\Delta \mathbf{q}, \lambda+\Delta \lambda)$ by solving the incremental equilibrium equation iteratively at a known solution point $(\mathbf{q}, \lambda)$ and the convergence of the solution is verified using the total equilibrium condition at the known solution point. In FEA, the total equilibrium condition at the known point is given by,

$$
\left[\mathbf{K}_{s}\right] \cdot \mathbf{q}+\lambda \mathbf{F}=0,
$$

where $\left[\mathbf{K}_{s}\right]$ is the secant stiffness (or simply stiffness) matrix for the system; and, the equilibrium at the unknown point is equivalently represented by the incremental equilibrium equation at the known point,

$$
\left[\mathbf{K}_{t}\right] \cdot \Delta \mathbf{q}-\Delta \lambda \mathbf{F}=0,
$$

where $\mathbf{q}$ and $\lambda$ are the nodal displacement vector and the load factor at the known solution point while $\Delta \mathbf{q}$ and $\Delta \lambda$ are their incremental values; $\mathbf{F}$ are the discretized reference nodal forces (typically as specified in the input for the problem); and $\left[\mathbf{K}_{t}\right]$ is the tangent stiffness matrix of the system.

\subsection{Iterative nonlinear solution}

In a nonlinear system, the incremental solution, (11), is sought in an iterative sense (e.g. Newton-Raphson iterations) and the total equilibrium condition, (10), is used to verify convergence of the incremental solution. In every increment, the new solution is sought by incrementing the load factor by a specified step as,

$$
\begin{aligned}
& \lambda_{n}^{1}=\lambda_{n-1}+\Delta \lambda_{n}^{1}, \\
& \mathbf{q}_{n}^{1}=\mathbf{q}_{n-1}+\Delta \mathbf{q}_{n}^{1},
\end{aligned}
$$

where, $(*)_{n}^{i}$ represents the quantity $(*)$ corresponding to the $i$ th iterative cycle during $n$th incremental solution and $(*)_{n}$ represents the converged quantity $(*)$ at the end of $n$th increment.

This step involves selection of appropriate initial load increment $\Delta \lambda_{n}^{1}$ for the first iterative cycle of the incremental solution. The choice of the initial increment should reflect the current degree of nonlinearity. If it is too large the solution converges slowly or may not converge at all. If it is too small, the solution becomes inefficient from a computer response point of view. Several strategies are presented in literature for automatic initialincrement-control based on convergence history (Crisfield 1981; Ramm 1981) and the so-called current stiffness parameter (Bergan et al 1978; Chan 1988). In this paper, the initial arc-length increment is chosen based on convergence history and the previous initial arc-length increment so that the cumulative displacements and the load level at the end of the first iteration are,

$$
\begin{aligned}
& \lambda_{n}^{1}=\lambda_{n-1} \pm \frac{\Delta \lambda_{n-1}^{1}\left(\mathbf{s}_{n-1} \cdot \mathbf{s}_{n-1}\right)^{1 / 2}}{\left(\mathbf{s}_{n} \cdot \mathbf{s}_{n}\right)^{1 / 2}}\left(\frac{I_{e}}{I_{n-1}}\right)^{\gamma} ; \\
& \mathbf{q}_{n}^{1}=\mathbf{q}_{n-1} \pm \frac{\Delta \lambda_{n-1}^{1}\left(\mathbf{s}_{n-1} \cdot \mathbf{s}_{n-1}\right)^{1 / 2}}{\left(\mathbf{s}_{n} \cdot \mathbf{s}_{n}\right)^{1 / 2}}\left(\frac{I_{e}}{I_{n-1}}\right)^{\gamma} \cdot \mathbf{s}_{n},
\end{aligned}
$$




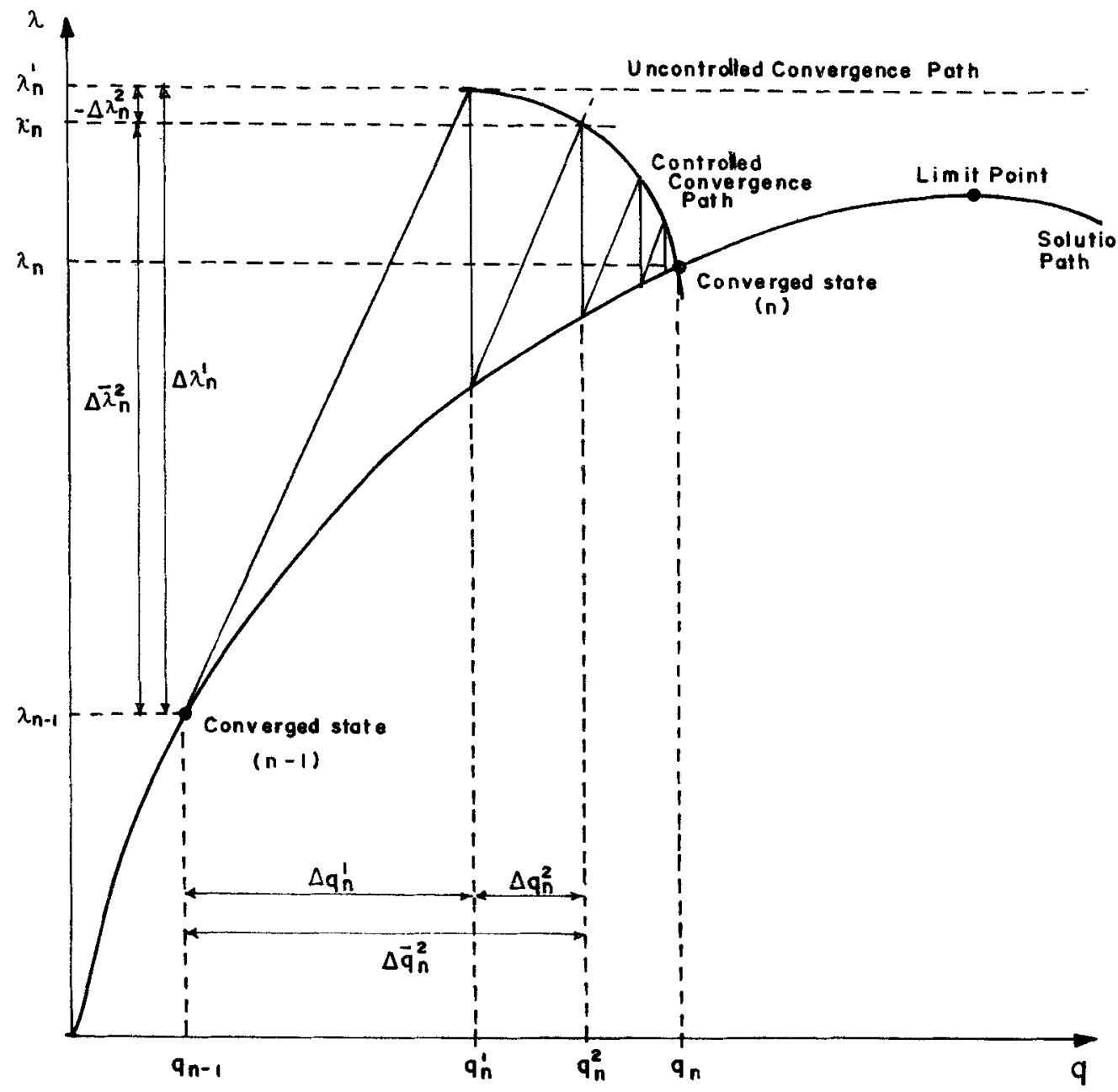

Figure 6. Incremental solution with controlled equilibrium iterations.

where, $\mathbf{s}_{n}$ is the reference solution corresponding the reference load vector $\mathbf{F}_{n}$ computed as $\mathbf{s}_{n}=\left[\mathbf{K}_{t}\right]^{-1} \mathbf{F}_{n}, I_{e}$ is the expected number of iterations for convergence in general, $I_{n-1}$ is the number of iterations taken for convergence in the previous incremental solution, and $\gamma$ is a parameter that takes a value from 0.5 to 1.0 .

Invariably, the above solution does not satisfy the total equilibrium condition (e.g. (10)) when the structural behaviour is nonlinear, and hence, additional iterative cycles are required to restore the equilibrium. In this paper, modified Newton-Raphson iterations are used for solving the incremental equilibrium equations, (11). It can be seen that the conventional iteration strategy at constant load increment exhibits low convergence rate and may not converge near a limit point. On the other hand, if the load increment is allowed to vary as in figure 6 , convergence rate is enhanced and the limit points could be traversed successfully since the solution is forced to converge along a constrained convergence path. Several equilibrium iteration control strategies - pure displacement control (Powell 
\& Simons 1981; Bergan \& Mollestad 1984), hybrid increment or arc-length control (Riks 1979, 1984; Wempner 1979; Crisfield 1981), minimum residual force norm (Bergan 1980), minimum residual displacement norm (Chan 1988), constant external work norm (Powell \& Simons 1981), and constant weighted response norm (Gierlinski \& Smith 1985) - have been proposed in literature with varied success.

Here, the most efficient strategy of all - arc-length continuation - is used for determining the load increment at each equilibrium iteration. Then, the constraint equation for computing the load increment during the current iteration $\left(\Delta \lambda_{n}^{i}\right)$ is,

$$
\left(\mathbf{q}_{n}^{i}-\mathbf{q}_{n-1}\right) \cdot\left(\mathbf{q}_{n}^{i}-\mathbf{q}_{n-1}\right)+\left(\lambda_{n}^{i}-\lambda_{n-1}\right)^{2} \mathbf{F}_{n} \cdot \mathbf{F}_{n}=\left(\Delta \lambda_{n}^{1}\right)^{2} \mathbf{s}_{n} \cdot \mathbf{s}_{n},
$$

where the current load parameter and displacement vector are expressed, respectively, as,

$$
\begin{aligned}
\lambda_{n}^{i} & =\lambda_{n}^{i-1}+\Delta \lambda_{n}^{i}, \\
\mathbf{q}_{n}^{i} & =\mathbf{q}_{n}^{i-1}+\Delta \mathbf{q}_{n}^{i} .
\end{aligned}
$$

The quadratic equation resulting from substituting (15) into (14) can be readily solved to compute the current load increment $\Delta_{n}^{i}$.

\subsection{Automated post-buckling path tracing}

Automated post-buckling involves: detection of possible unstable behaviour and the choice of appropriate initial-increment direction so that the solution path is not retraced; classification of the detected unstable behaviour of the structure; and branch-switching and computation of the post-buckling solution(s).

In the present work, a singularity point is detected during the current increment if the determinant of the tangent stiffness matrix $\left(\left\|\mathbf{K}_{t}\right\|_{n}\right)$ changes its sign. Once the tangent stiffness matrix is decomposed as, $\left(\mathbf{K}_{t}\right)_{n}=(\mathbf{L} \cdot \mathbf{D} \cdot \mathbf{L})_{n}$, we have,

$$
\left\|\mathbf{K}_{t}\right\|_{n}=\prod_{l=1}^{n d o f}\left(D_{l l}\right)_{n} .
$$

Two methods are known for classifying the detected singularities as limit points and bifurcation points: the first based on the current stiffness parameter (Brendel \& Ramm 1980) and the second on the properties of the so-called generalised deflection (Huang \& Atluri 1995). Here, the identified instability points are classified as limit points or bifurcation points using the later strategy.

If the identified instability points are limit points (snap-through/snap-back buckling), the arc-length controlled equilibrium iterations will successfully trace the post-buckling solution path. Several methods have been proposed for automated branch-switching in post-buckling structural analysis - e.g. perturbation method (Wagner \& Wriggers 1988) and linearised asymptotic solution technique (Huang \& Atluri 1995). Normally, if the instability point is a bifurcation point, its location is computed and then, based on an eigenvalue solution, appropriate perturbation is applied to follow the desired post-buckling branch in an asymptotic linear sense.

The nonlinear fundamental state between two solution points $n-1$ and $n$ in the neighbourhood of a bifurcation point is linearised to obtain the asymptotic solution (Koiter 1945; 
Huang \& Atluri 1995). After linearising the nonlinear path between $n-1$ and $n$, consider an adjacent (asymptotic) state $\tilde{\mathbf{q}}_{n}^{i}$ near the fundamental state $\mathbf{q}_{n}^{i}$ :

$$
\tilde{\mathbf{q}}_{n}^{k}=\mathbf{q}_{n}^{k}+\eta_{k}=\mathbf{q}_{n}^{k-1}+\tilde{\lambda}_{k} \Delta \mathbf{q}_{n}^{k}+\eta_{k} .
$$

Substituting (17) into (10), rearranging the tangent stiffness components that are independent, linearly dependent and quadratically dependent on the linearised load parameter $\tilde{\lambda}_{k}=\left(\lambda_{n}^{k}-\lambda_{n-1}\right) /\left(\lambda_{n}-\lambda_{n-1}\right)$ as $\mathbf{K}_{0 n}, \mathbf{K}_{L n}, \mathbf{K}_{N n}$ respectively, and applying the condition of buckling at load level $\tilde{\lambda}_{k}$, we get the following iterative equations for the eigenvalue problem:

$$
\mathbf{K}_{0 n} \cdot \boldsymbol{\eta}_{k}=\tilde{\lambda}_{k}\left(-\mathbf{K}_{L n}-\tilde{\lambda}_{k-1} \mathbf{K}_{N n}\right) \eta_{k}
$$

where $\tilde{\lambda}_{k-1}$ is the approximate eigenvalue in the previous iteration. The approximate critical buckling load factor $\tilde{\lambda}_{c r}$ obtained can be used to compute the eigenvector $\hat{\eta}$ which can be normalized using the following condition:

$$
\hat{\boldsymbol{\eta}} \cdot \mathbf{K}_{0 n} \cdot \hat{\boldsymbol{\eta}}=1 \text {. }
$$

Note that, since the problem is linearised, the solution understandably consumes much less computer time.

A linear combination of the eigenvector $\hat{\eta}$ and its orthogonal counterpart $\rho$ is used to excite an internal perturbation in the nonlinear fundamental solution path so as to switch to the desired secondary post-buckling paths (Huang \& Atluri 1995).

Finally, several convergence criteria - based on several residual displacement and/or residual force norms - are available in literature. Here both displacement and force based norms are used for verifying convergence of nonlinear solutions. One may refer to Naganarayana (1995) for a unified presentation of different strategies involved in a completely automated post-buckling solution for finite element analysis of geometrically nonlinear structures.

\section{NONCAT: NONlinear Computational Analysis Tool for structural applications}

A finite element software is developed for general nonlinear analysis of stiffened delaminated structures based on the formulation presented in this paper (Huang et al 1995) aided by simple pre- and post-processing. Figure 7 shows a schematic diagram of the software organisation.

The element library incorporates shear-flexible curved 2-noded beam (BEAM2) and 3-noded quasi-conforming shell (SHELL3) elements. The problems of locking are alleviated by using reduced integration for the membrane strain energy. The core finite element package is supported by general nonlinear solution tools. The solution module incorporates the Gauss elimination in a cycle of Newton-Raphson iterations. The load incrementation is automated using the arc-length continuation technique for optimal convergence. Detection and classification of the instability points are also automated, based on certain specific properties of the generalised deflection and some simple heuristic rules. The solution automatically switches the path based on asymptotic post-buckling theory if the detected instability is found to be of the bifurcation type. Finally, the solution is tested for convergence. If the solution does not meet the convergence requirements, the solution is sought 


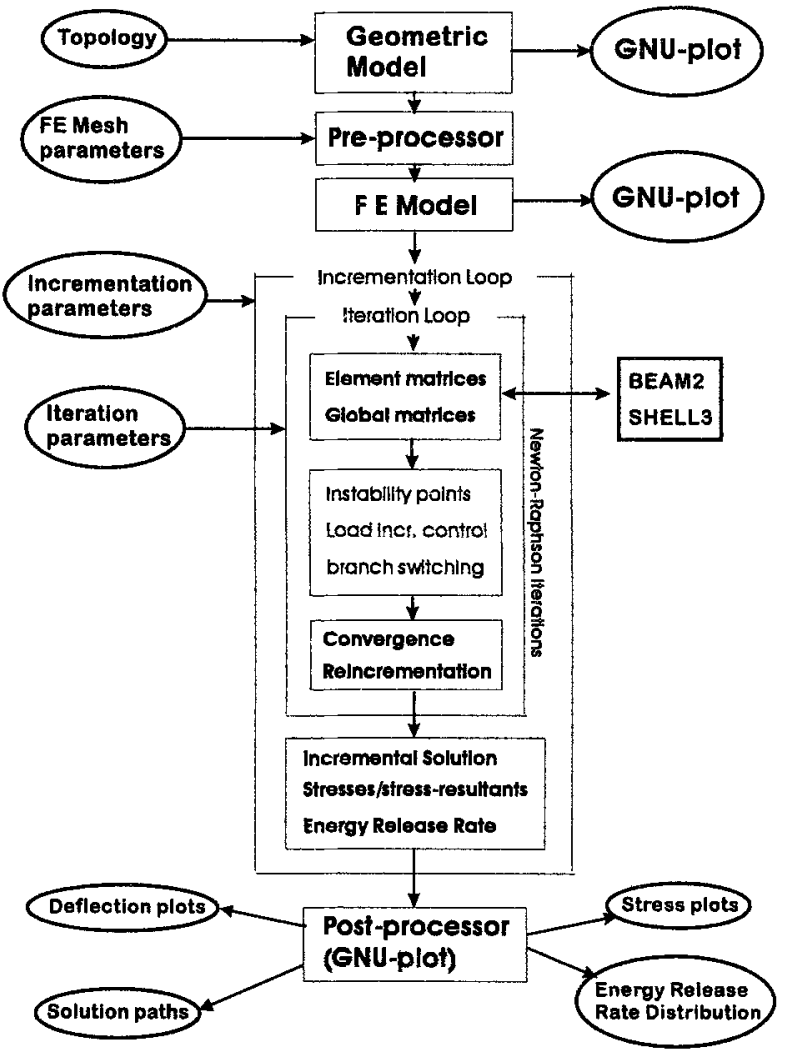

Figure 7. NONCATS: NONlinear Computational Analysis Tool for Structural applications.

again with new appropriate initial load increments. Once the incremental solution has converged, the displacements are processed to get stresses, stress resultants and displacement gradients which are in turn used for computing the energy release rate distribution along the delamination front.

Simple pre- and post-processing is provided using the graphical user interface GNUplot. Topological modelling is done based on user-fed geometric data for the substructures and their connectivity. A finite element mesh generator is developed for finite element modelling in substructure level. The multi-point constraints satisfying the conditions of Reissner-Mindlin plate theory are incorporated to model the delamination front as well as stiffener-shell joints. The geometric and finite element models are interfaced with the GNU for graphical presentation. The results at the end of each load increment - nodal displacements, solution paths, stresses/stress-resultants, and energy release rate distribution along the delamination front - are again interfaced with GNU for graphical presentation.

\section{Numerical experiments: Coupled failure processes in delaminated structures}

In this section, the proposed finite element analysis strategy is validated using an available analytical solution for local buckling and delamination growth. Several numerical experiments have been conducted to establish the influence of several structural parameters on post-buckling structural behaviour and delamination growth (Naganarayana \& Atluri 1995c; Naganarayana \& Huang 1995; Naganarayana et al 1995). Here the model is first 
validated with reference to an available analytical solution and later a few salient numerical examples are considered to demonstrate the coupled failure processes - detrimental interaction between the geometric failure (local/global laminate buckling) and the material failure (delamination growth) - in a delaminated stiffened/composite structure.

\subsection{Model validation}

Here, we shall validate the finite element solutions with reference to an available analytical solution (Evans \& Hutchinson 1984) using an isotropic square plate of edge length $L$ with a central elliptic delamination. The plate is subjected to biaxial compressive loads and its boundary is assumed to be clamped against out-of-plane deformations. One quarter of the plate is modelled for the analysis by imposing appropriate symmetry conditions. 264 shell elements are used for the nondelaminated plate and 192 elements each are used for the delaminate and base plates. The reference applied biaxial compressive loads are assumed to be of unit intensity $\left(F_{I}=1.0\right)$ and the equilibrium equations are solved at each load step for an applied load $F=\lambda F_{I}$, where $\lambda$ is the corresponding load factor.

The structure is assumed to be isotropic with Young's modulus $E=6500$ and Poisson's ratio $v=0.3$. The laminate thickness is chosen as $t_{1}=0.05 \mathrm{~L}$. The numerical experiment is conducted for a near-surface circular delamination with $t_{2} / t_{1}=0.01, a / b=1.0$ and $a / L=0.3$. Assuming that the base plate and the nondelaminated plates are infinitely stiff when compared to the delaminated plate, the delaminated plate can be considered a clamped circular plate under the same radial compressive stress (Evans \& Hutchinson 1984). Then, the buckling strength of the delaminate plate $\left(\sigma_{c}\left(\equiv \lambda_{c r}^{l} F_{I} / t_{1}\right)\right)$ is given by

$$
\sigma_{c}=1.2233\left(\frac{E}{1-v^{2}}\right)\left(\frac{t_{2}}{a}\right)^{2} .
$$

The local buckling strength of the delaminate plate obtained from the finite element analysis compares very accurately with the analytical estimate, (20) as shown in figure 8a.

Further, assuming that the post-buckling deformation is axisymmetric and nearly linear in the neighbourhood of local buckling point, the pointwise energy release rate is given by (Evans \& Hutchinson 1984),

$$
\mathcal{G}_{r b}(\Gamma)=\frac{\left(1-v^{2}\right) t_{2}}{(1.8285+v) E}\left(\sigma_{0}^{2}-\sigma_{c}^{2}\right),
$$

where $\sigma_{0}\left(\equiv \lambda F_{I} / t_{1}\right)$ is the actual stress level at which the energy release is being computed.

The ratio, $\mathcal{G}_{F E} / \mathcal{G}_{r b}$, is plotted along the delamination periphery $\left(\theta=0^{\circ}-90^{\circ}\right.$ for the quarter circle) in figure $8 \mathrm{~b}$ for the case of a very thin delaminate configuration $\left(t_{2} / t_{1}=\right.$ 0.01 ). It can be observed that, $\mathcal{G}_{F E}$ is close to $\mathcal{G}_{r b}$ when the post-buckling loads are in the close vicinity of local buckling point (i.e. $\lambda^{*} \equiv \lambda / \lambda_{c r}^{l} \simeq 1.0$ ). However, $\mathcal{G}_{r b}$ is underestimated when compared to $\mathcal{G}_{F E}$ even when $\lambda \simeq \lambda_{c r}^{l}$. This is because, in the present problem, though the delaminate plate is very thin when compared to the total laminate thickness $\left(t_{2} / t_{1}=0.01\right)$, the base plate is flexible as opposed to the rigid base as considered in Evans \& Hutchinson (1984).

The laminate is also thin when compared to its edge length, $L\left(t_{1} / L=0.05\right)$. Hence, the finite element model represents reasonably flexible laminate and base plates as well. 

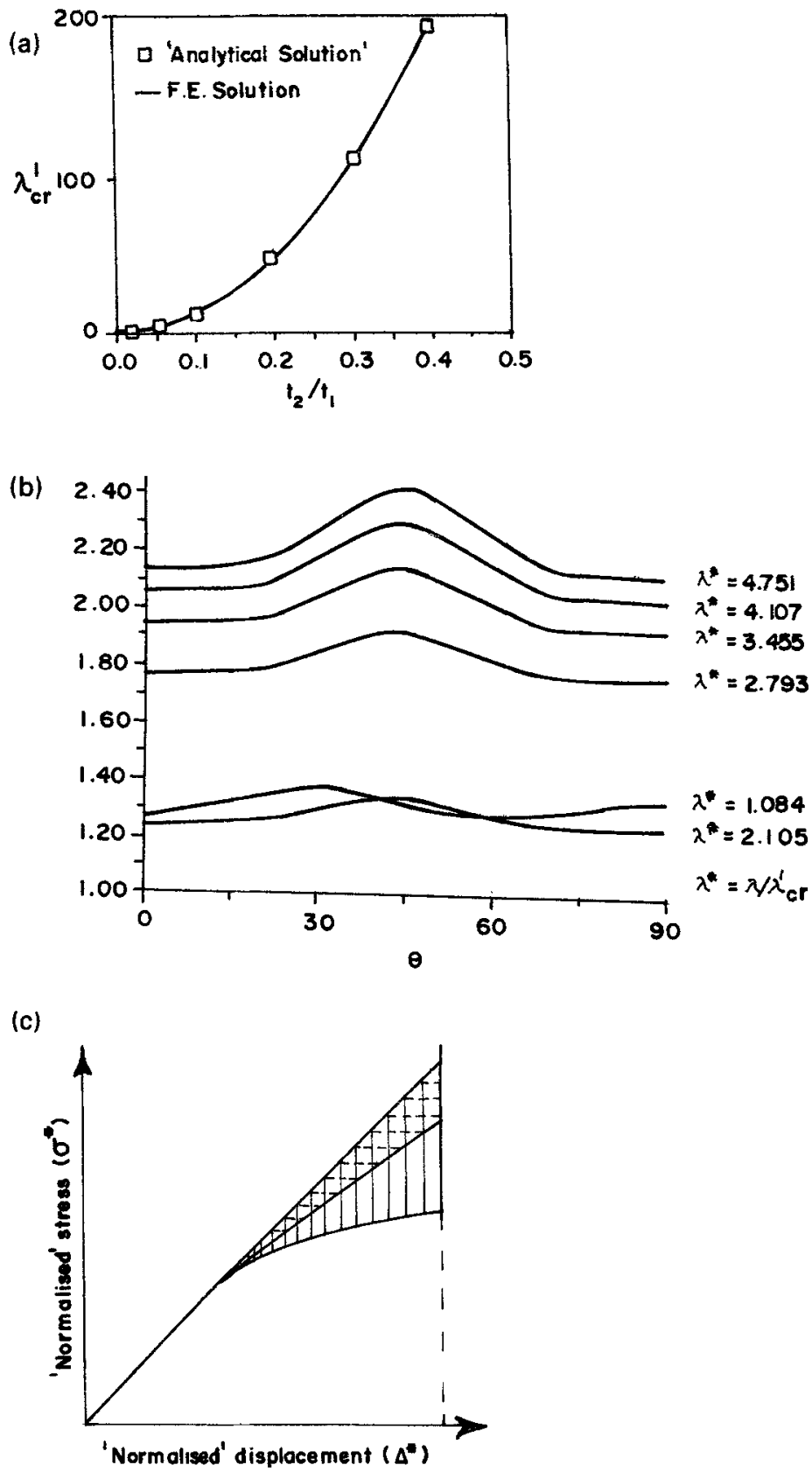

Figure 8. Model validation: Circular thin delaminate in square isotropic plate - (a) Critical local buckling strength: Comparison of FE solution with an analytical solution; (b) pointwise energy release rate distribution along delamination edge: Comparison of FE solution with an analytical solution; (c) effect of 'quasi-linear post-buckling behaviour for delaminate on energy release rate. 
The deviation increases as the buckling load increases beyond its critical value for local buckling of the delaminate plate. This is because, the analytical solution, (21), is based on the assumption of a quasi-linear post-buckling behaviour for the delaminate plate. But, in practice, particularly when the laminate is thin, post-buckling behaviour of the delaminate plate is highly nonlinear. Accordingly, much higher energy-release-rates are expected when compared to $\mathcal{G}_{r b}$ as shown in figure 8c. Note that, in figure $8 \mathrm{c}$, actual stress $(\sigma)$ and displacement $(\Delta)$ are 'normalised' by the critical stress $\sigma_{c}$ and the associated critical inward radial displacement $\Delta_{c}$ respectively.

\subsection{Laminated shell with elliptic delamination}

In this section, a cylindrical laminated shell of edge length $L$ with a central elliptic delamination (near the inner shell surface) under axial compressive loads is considered. The shell is assumed to be constituted with 32 orthotropic laminae of equal thickness stacked in a symmetric fashion: $(0 / 90 / 45 /-45)_{s}$. The shell thickness is assumed as $t_{1}=0.05 L$. The delamination configuration is fixed as: $a / L=0.3 ; a / b=1.5$; and $t_{2} / t_{1}=1 / 32$. The major axis of the delamination is oriented parallel to the shell axis. The material properties for each layer are: $E_{1}=208000 ; E_{2}=26000 ; v_{12}=v_{13}=v_{23}=0.16$; $G_{12}=G_{13}=G_{23}=7500$. The reference load intensity is assumed to be unity. The shell boundary is clamped against out-of-plane deformation. Keeping the edge-length constant ( $R \psi=L$, where $R$ is the radius of curvature and $\psi$ is the angle included), the shell curvature is changed for studying its effects on the buckling and delamination growth behaviour of the structure.

The post-buckling delaminate and base shell deformation (transverse deflection $w$ at centroid) is depicted for typical shell curvatures in figures $9 a-d$. It can be observed that the critical load factor for local delaminate buckling increases as curvature increases in a linear sense (figure 9e). The global buckling strength of the structure also increases as the shell curvature increases; however, due to the presence of the delamination, the structure exhibits reduced global buckling strength (results not shown). The maximum and average pointwise energy release rates are presented for varying load factor for typical shell curvatures in figures $10 \mathrm{a}-\mathrm{d}$. It can be seen that the energy release rate decreases as the shell curvature increases (figure 10e). Thus local delaminate buckling and delamination growth are delayed in shells when compared to plates.

\subsection{Stiffened laminated plate with elliptic delamination}

In this section, we shall consider a laminated composite square plate of edge length $L$ with 32 orthotropic laminae of equal thickness and stacked symmetrically: $(0 / 90 / 45 /-45)_{s}$. The plate thickness is assumed to be $t_{1}=0.025 \mathrm{~L}$. The central elliptic delamination configuration is fixed as: $a / L=0.15 ; a / b=1.50$; and $t_{2} / t_{1}=1 / 32$. The material properties for each layer are taken as in the previous example. The plate is stiffened in both directions in a symmetric fashion as shown in figure 11. The distance between the stiffeners is assumed as $d=L / 2$. The sectional properties of each stiffener in axial, inplane flexure, out-of-plane flexure, twisting, and transverse shear deformations are respectively: $E A=0.104 \times 10^{9}, E I_{x x}=0.8667 \times 10^{9}, E I_{y y}=0.2167 \times 10^{9}, G J=0.39063 \times 10^{7}$, 

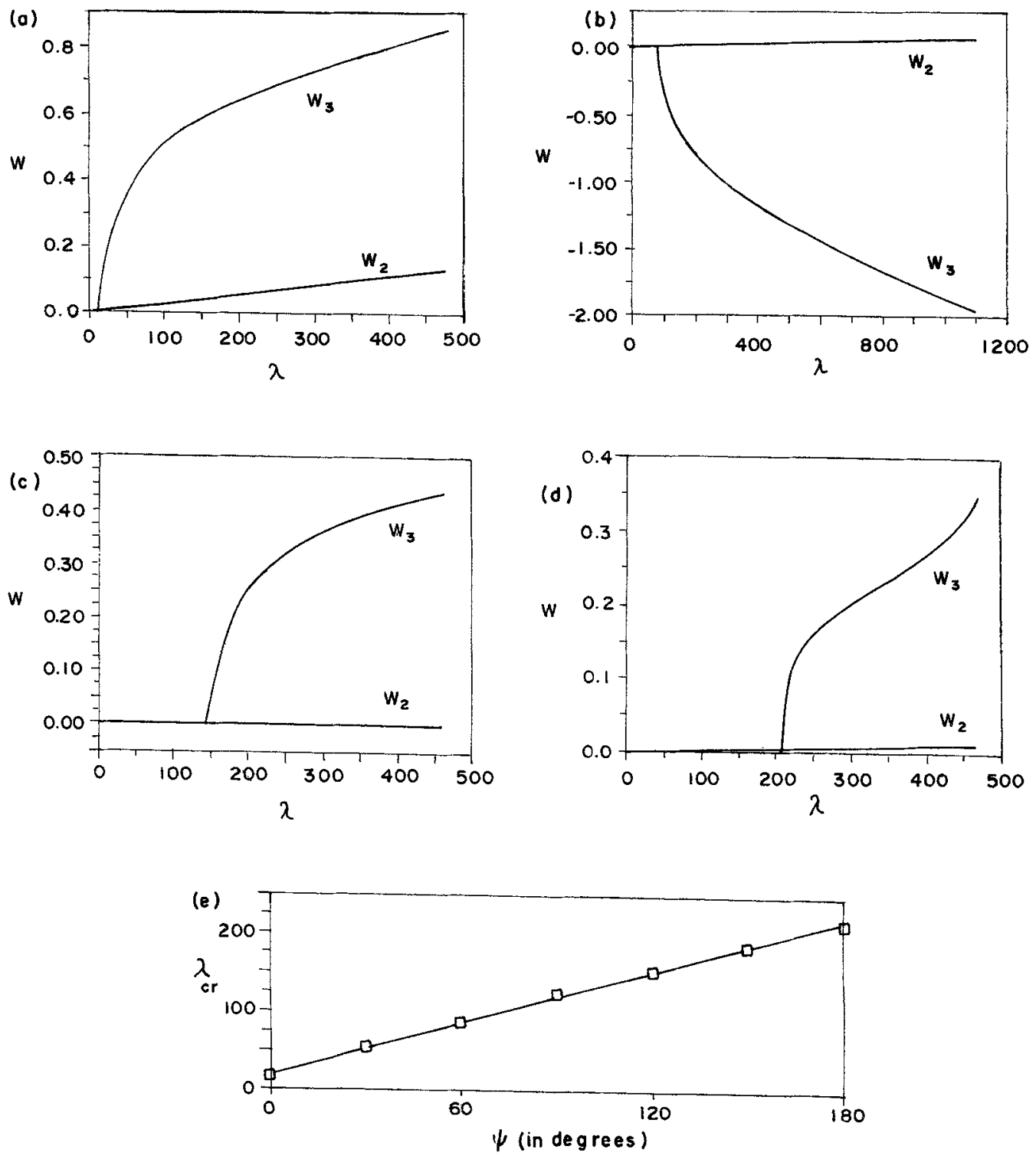

Figure 9. Cylindrical shell: Effects of shell curvature on buckling/post-buckling performance: $\theta=0^{\circ}(\mathbf{a}), 60^{\circ}$ (b), $120^{\circ}$ (c) and $180^{\circ}$ (d); (e) critical local buckling load factor.

and $G A=0.375 \times 10^{6}$. The reference applied biaxial compressive loads are assumed to be of unit intensity. The plate boundary is clamped against out-of-plane deformation. Considering the symmetry of the problem, a quarter of the plate is modelled. 20 beam elements are used to model each stiffener; while 92 shell elements each are used to model the delaminate and the base plates, 264 shell elements are used to model the nondelaminated plate between the stiffeners, and 128 shell elements are used to model the rest of the plate. The numerical experiments are conducted for the plate with and without stiffeners at different eccentricities: 

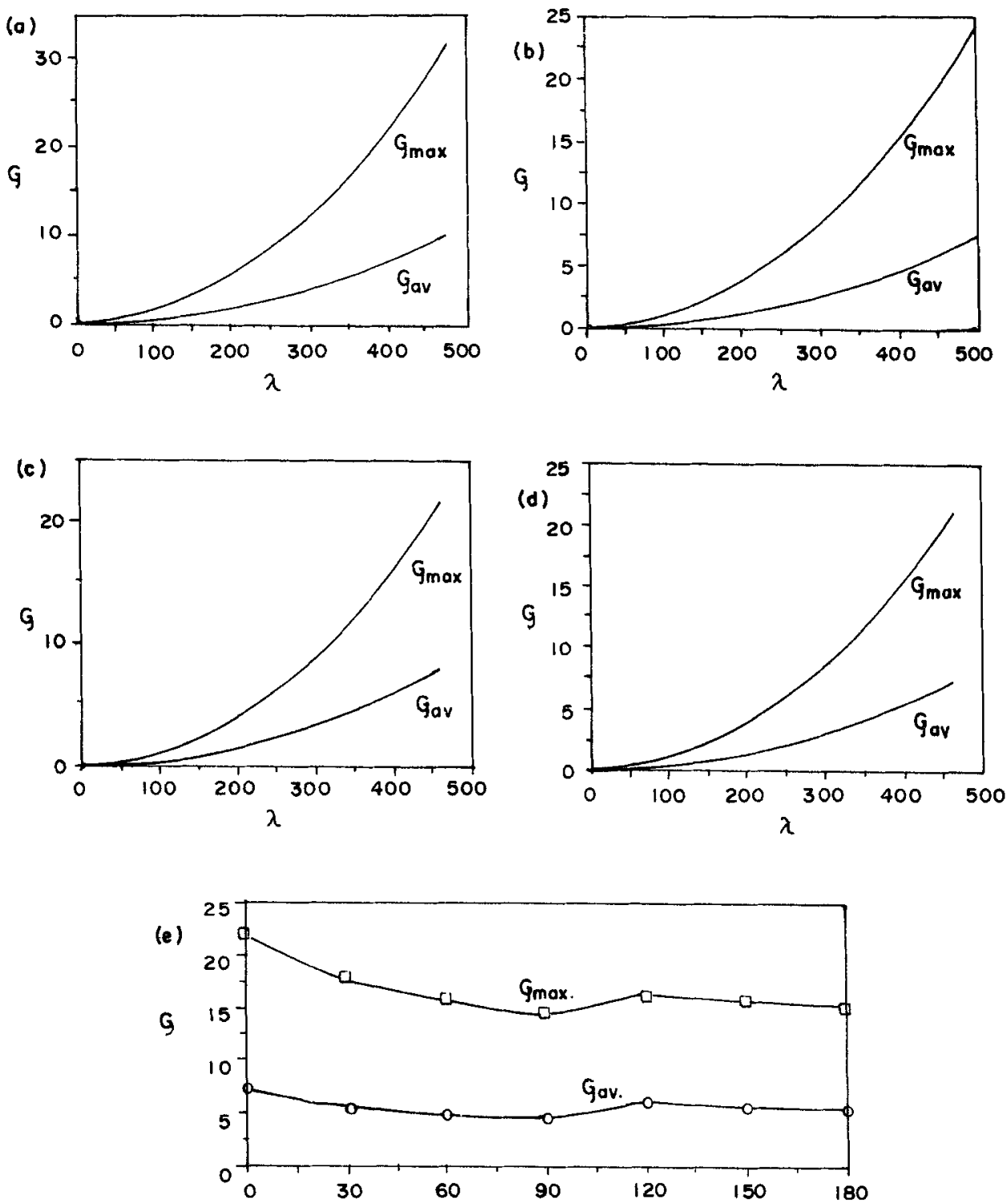

Figure 10. Cylindrical shell: Effects of shell curvature on average and maximum energy release rates $-\theta=0^{\circ}(\mathbf{a}), 60^{\circ}(\mathrm{b}), 120^{\circ}$ (c) and $180^{\circ}$ (d); (e) average and maximum energy release rates.

Case-a: plate with no stiffeners,

Case-b: plate with non-eccentric stiffeners, $e=0$,

Case-c: plate with stiffeners on opposite side of the delamination, $e=-10$,

Case-d: plate with stiffeners on the same side of the delamination, $e=+10$.

The post-buckling deflections of the delaminate and the base plates are depicted in figure 12. The delaminate buckling strength increases with the inclusion of a stiffener. It may be noted that stiffener's with zero eccentricity with reference to the plate provide maximum delay in the delaminate plate buckling. It is interesting to note that stiffeners do 

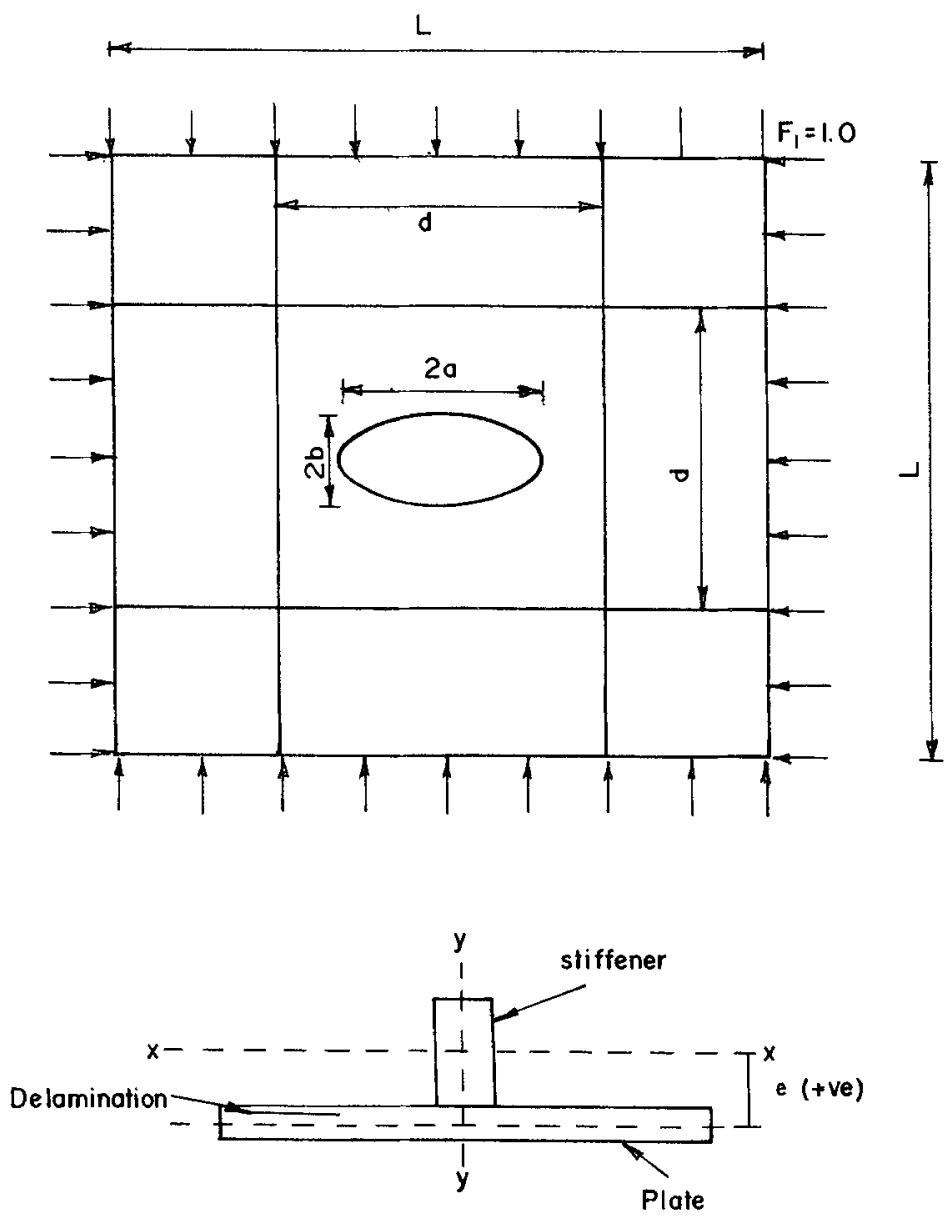

Figure 11. Stiffened laminated plate with central elliptic delamination.

not appreciably increase local buckling strength when the delamination is located in the opposite side when compared to the stiffeners (case-c).

The average and maximum pointwise energy release rates are presented for the different cases in figure 13. It can be observed that noneccentric stiffeners (case-b) considerably decrease both the average and the maximum energy release rates for a given load. However, introduction of eccentric stiffeners (case-c and case-d) lead to appreciable increase in the average energy release rate for a given load. Though stiffeners on the same side as the delamination (case-d) slightly decrease the maximum energy release rate, stiffeners on the opposite side when compared to the location of the delamination (case-c) increase the maximum energy release rate considerably. Thus, noneccentric stiffeners (case-b) delay the delamination growth appreciably. On the other hand, eccentric stiffeners (case-c and case-d) may lead to considerably accelerated delamination growth. Thus from both geometric and material failure points of view, non-eccentric stiffeners are preferable while reinforcing a delaminated structure. However, in most aerospace applications, the stiffeners are located internally for aerodynamic requirements, and the external surface is highly susceptible to loads causing delaminations. Thus, results for case-c appear to be the most critical from practical considerations. Similar 

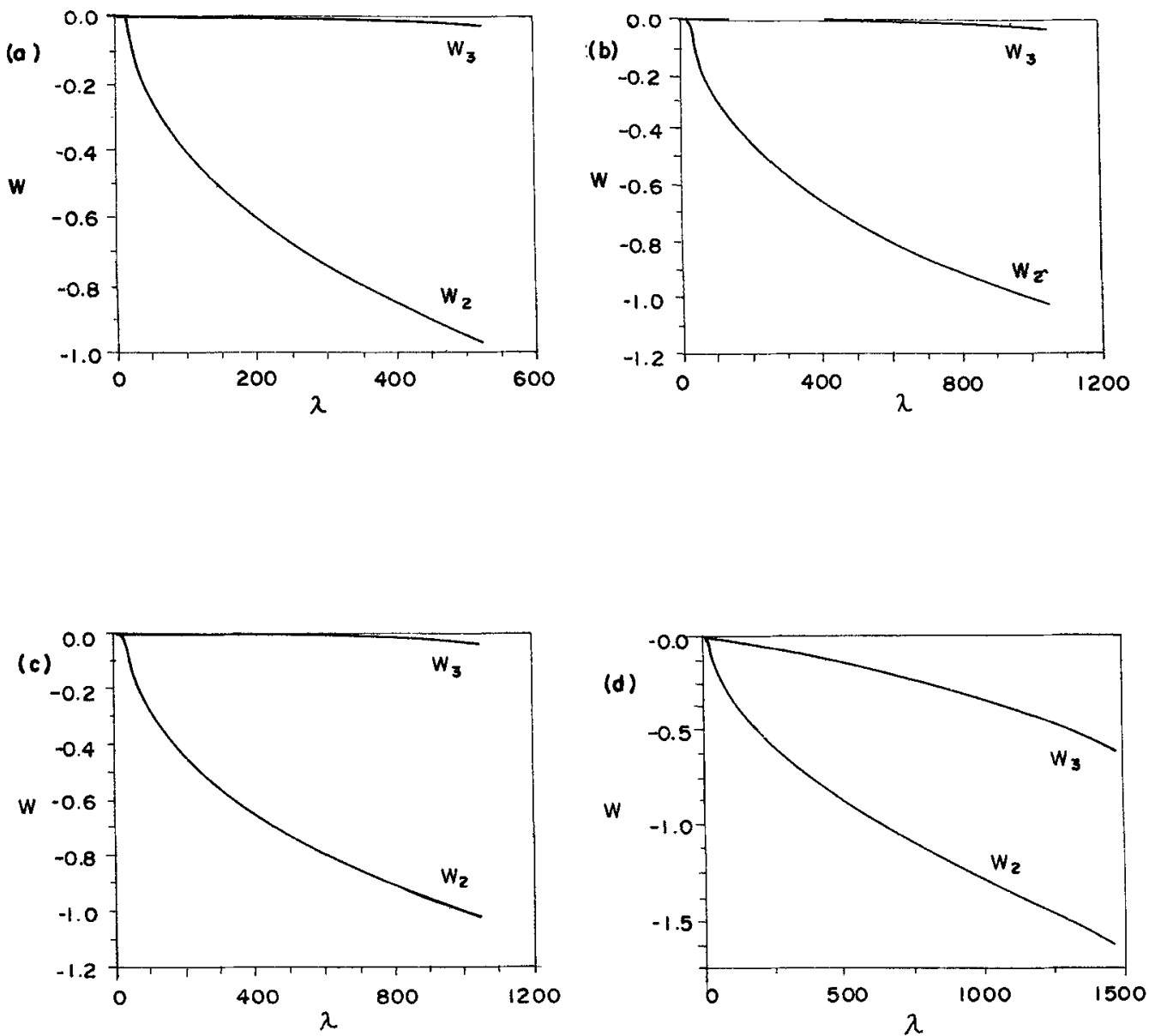

Figure 12. Stiffened laminated plate: Post-buckling delaminate and base deflections: (a) No stiffeners; (b) symmetric stiffeners $(e=0)$; (c) eccentric stiffeners $(e=-10)$; (d) eccentric stiffeners $(e=+10)$.

behaviour was observed with reference to stiffened laminated shells (results not shown).

\section{Conclusions}

The laminated and stiffened structures are particularly prone to interlaminar debonding (delamination) type of failures since the interlaminar bond strength is much less when compared to in-plane laminar strength. The delaminations are very dangerous since they drastically reduce laminate strength, particularly its buckling strength. In addition, the delaminations grow under operating conditions, particularly compressive loads, further reducing the structural strength leading to fatal structural failure. In this paper, a complete methodology is presented for analysing delaminated structures for their residual strength and the possible growth of the delamination (particularly under compressive loading) which could be used for optimal structural design as well. 

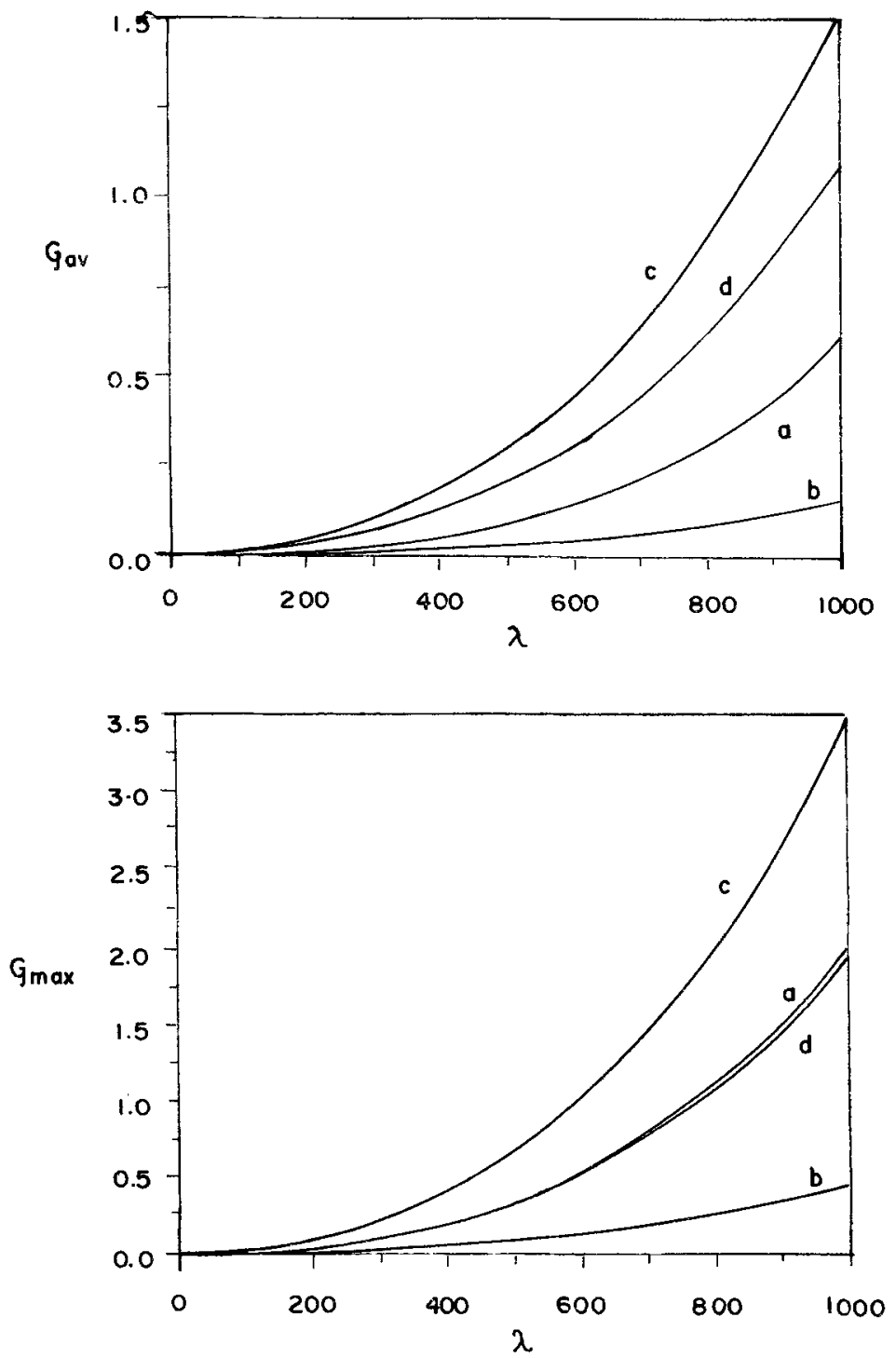

Figure 13. Stiffened laminated plate: Average and maximum energy release rate.

A robust finite element method is presented for modelling the delaminated structures, for obtaining accurate structural response and for predicting the delamination growth in terms of pointwise energy release rate (figure 7). This program can be enhanced into a powerful general purpose software for modelling and analysing failure in general stiffened composite structure by combining the existing library of robust finite elements (e.g. FEPACS: version-1.0, Prathap \& Naganarayana 1991), solution capabilities (e.g. FEPACS: version-2.1, Prathap et al 1994), NONCAT (Huang et al 1995), and the advanced modelling software for structural and finite element modelling with special modules for modelling damage, repair and damage control mechanisms, for predicting damage initiation and growth, and for designing/analysing appropriate repair/damagecontrol mechanisms, and with expert advisor systems for problem modelling and for 


\section{Expert Assisted Pre-Processor}
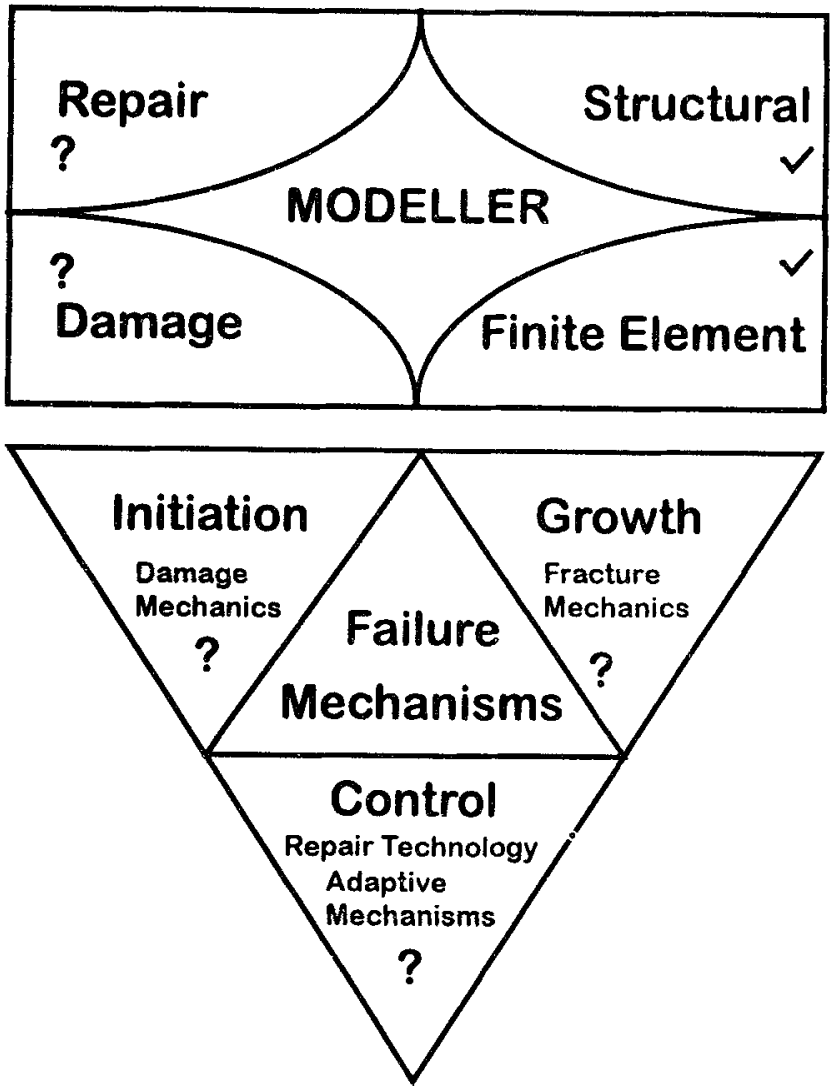

Field-consistent Variationally Correct 1-, 2-, and 3-D Linear and Quadratic Displacement type Elements for

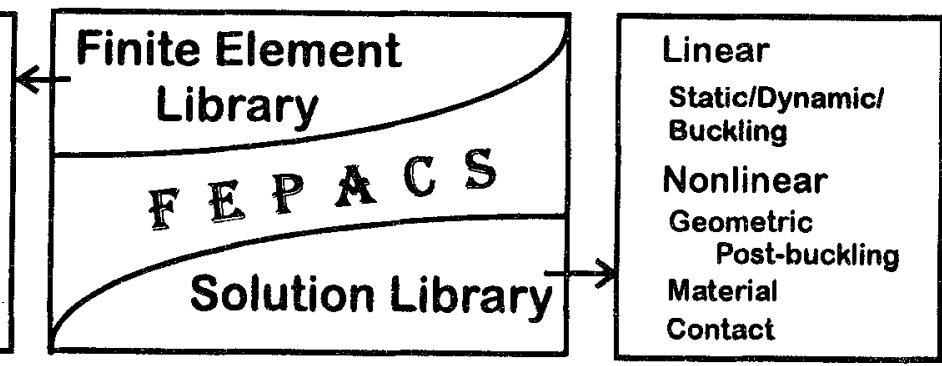

\section{Expert Assisted Post-Processor}

Figure 14. A general purpose expert-aided environment for failure prediction/assessment and designing damage repair/control mechanisms: A desirable infrastructure. 
processing the results as shown in figure 14 using the guidelines outlined in this paper.

Curved shear-flexible 2-noded stiffener and 3-noded shell elements free of membrane locking and nonlinear locking are presented. An automated general nonlinear solution strategy that can successfully pass the instability points of any kind is incorporated such that the multiple post-buckling solution paths that can exist in delaminated structures and their interaction could be accurately computed. Arc-length continuation is used for passing the instability points and for optimal convergence rate. The instability points are detected and classified based on the specific properties of the tangent stiffness and the generalised deflection. If the detected instability is of bifurcation type, branch-switching (to follow the desired secondary solution paths) is achieved effectively using a simple and cost-effective method based on an asymptotic linearised eigenvalue solution at the instability point. Finally, the displacements and displacement gradients are post-processed to compute stresses and stress resultants at element centroids and pointwise energy release rate distribution along the delamination front. The 3 -dimensional $J$-integral is used to derive the pointwise energy release rate as a function of the stress-resultants and displacement gradients in the neighbourhood of the delamination front, and the jump in strain energy density across the delamination edge.

Unlike the conventional methods of 3-dimensional analysis and/or global-local analysis, the method presented in this paper is simple and cost-effective, particularly with reference to the nonlinear post-buckling structural behaviour when the delaminated structures are subjected to compressive loads. The methodology also provides capability to capture multiple buckling modes (local, intermediate and global); to predict delamination growth in pre-buckling, post-buckling regimes; and to compute the interaction between the geometric and material failures (buckling and delamination growth, in this case) effectively. Some typical numerical examples are critically examined to validate the proposed 2-dimensional computational model and to demonstrate the coupled geometric and material failure mechanisms in delaminated composite structures.

The authors acknowledge the grants-in-aid support by FAA to the Center of Excellence for Computational Modeling of Aircraft Structures, Georgia Institute of Technology. The first author also acknowledges the Indo-US Science and Technology Fellowship under the auspices of the Department of Science and Technology, India and the US Agency for International Development, USA.

\section{References}

Atluri S N (ed.) 1986 Energetic approaches and path independent integrals in fracture mechanics. Computational methods in mechanics of fracture (Amsterdam: North-Holland)

Babu C R, Prathap G 1988 A linear thick curved beam element. Int. J. Numer. Methods Eng. 23: $1313-1328$

Barlow J 1976 Optimal stress locations in finite element models. Int. J. Numer. Methods Eng. 10: 243-251 
Bergan P G 1980 Solution algorithms for nonlinear structural problems. Comput. Struct. 12: 497-509

Bergan P G, Mollestad E 1984 Static and dynamic solution strategies in nonlinear analysis. em Numerical methods for nonlinear problems (ed.) C Taylor, (Swansea: Pineridge) pp. 3-17

Bergan P G, Horrigmoe B, Krakeland B, Soreide T H 1978 Solution techniques as applied to a nonlinear finite element problem. Int. J. Numer. Methods. Eng. 12: 1677-1696

Bottega W J, Maewal A 1983 Delamination buckling and growth in laminates. J. Appl. Mech. 50: 184-189

Brendel B, Ramm R 1980 Linear and nonlinear stability analysis of cylindrical shells. Comput. Struct. 12: 549-558

Chai H, Babcock C D, Knauss W G 1981 One-dimensional modeling of failure in laminated plates by delamination buckling. Int. J. Solids Struct. 17: 1069-1083

Chan S L 1988 Geometric and material nonlinear analysis of beam-columns and frames using the minimum residual displacement method. Int. J. Numer. Methods Eng. 26: 2657-2669

Crisfield M A 1981 A fast incremental/iterative solution procedure that handles "snap-through". Comput. Struct. 13: 55-62

Evans A G, Hutchinson J W 1984 On the mechanics of delamination and spalling in compressed film. Int. J. Solids Struct. 20: 455-466

Flanagan G 1988 Two-dimensional delamination growth in composite laminates under compression loading. Eighth Conf. on Composite Materials - Testing and Design (ed.) J D Whitcomb, ASTM STP 972 (Philadelphia: Am. Soc. Testing Mater.) pp. 180-190

Gierlinski J T, Smith T R G 1985 A variable load iteration procedure for thin-walled structures. Comput. Struct. 21: 1085-1094

Gilletta D 1988 Theories non lineaires des stratifies composites en compression avee delaminage. Rapport technique ONERA

Huang B-Z, Atluri S N 1995 A simple method to follow post-buckling paths in finite element analysis. Comput. Struct. 57: 477-489

Huang B-Z, Shenoy V B, Atluri S N 1994 A quasi-conforming triangular laminated composite shell element based on a refined first order theory. Comput. Mech. 13: 295-314

Huang B-Z, Naganarayana B P, Atluri S N 1995 NONCAT - A NONlinear Computational Analysis Tool for analysis of delaminated composite structures. Computational Modeling of Aircraft Structures, CMAS Symposium'95, Somerset, NJ

Kachanov L M 1976 Separation failure of composite materials. Polymer Mech. 5: 918922

Koiter W T 1945 On the stability of elastic equilibrium (Amsterdam: Polytechnic Institute Delft, H J Paris) (Also NASA TT F-10.833, 1967; and AFFDL-TR-70-25, 1970)

Naganarayana B P 1995 Incremental iterative strategies for automated post-buckling analysis. Comput. Struct. (in press)

Naganarayana B P, Atluri S N 1995a Equivalent domain integral for delamination growth estimation. ICES '95, Hawaii

Naganarayana B P, Atluri S N 1995b Energy release rate evaluation for delamination growth prediction in a multi-plate model of a laminate composite. Comput. Mech. 15: 443-459

Naganarayana B P, Atluri S N 1995c Strength reduction and delamination growth in thin and thick composite plates under compressive loading. Comput. Mech. 16: 170-189

Naganarayana B P, Huang B-Z 1995 Multi-domain modeling of delaminated stiffened composite structures. Symp. on Advanced Engineering Sciences, New Orleans

Naganarayana B P, Prathap G P 1996 Locking in a geometrically nonlinear beam element. AIAA $J$. (accepted) 
Naganarayana B P, Huang B-Z, Atluri S N 1995 Multi-domain modeling and analysis of delaminated stiffened composite structures. AIAA J. (in press)

Nikishkov G P, Atluri S N 1987a Calculation of fracture mechanics parameters for an arbitrary 3-dimensional crack by equivalent domain integral method. Int. J. Numer. Methods Eng. 24: 1801-1822

Nikishkov G P, Atluri S N 1987b An equivalent domain integral method for computing crack tip parameters in non-elastic thermo-mechanical fracture. Eng. Fracture Mech. 26: 851-867

Nishioka T, Atluri S N 1981 Analytical solutions for embedded cracks and finite element alternating method for elliptical surface cracks subjected to arbitrary loadings. Eng. Fracture Mech. 17: $247-268$

Parks D M 1974 A stiffness derivative finite element technique for determination of elastic crack tip stress intensity factors. Int. J. Fracture Mech. 10: 487-502

Powell G, Simons J 1981 Improved iteration strategy for nonlinear structures. Int. J. Numer. Methods. Eng. 17: 1455-1467

Prathap G 1993 The finite element method in structural mechanics (Dordrecht: Kluwer Academic Publications)

Prathap G, Naganarayana B P 1991 FEPACS, A finite element package for analysis of composite structures. PD ST 9139, National Aerospace Laboratories, Bangalore

Prathap G, Naganarayana B P, Somashekar B R 1994 FEPACS (Version 2.0); Finite element package for analysis of composite structures - Theoretical manual. PD ST 9405, National Aerospace Laboratories, Bangalore

Ramm E 1981 Strategies for tracing the nonlinear response near limit points. Nonlinear finite element analysis in structural mechanics (eds) W Wunderlich et al (Berlin: Springer-Verlag) pp. 63-89

Rice J R 1968 A path independent integral and approximate analysis of strain concentration by notches and cracks. J. Appl. Mech. ASME 35: 379-386

Riks E 1979 An incremental approach to the solution of snapping and buckling problems. Int. $J$. Solids Struct. 15: 529-551

Riks E 1984 Some computational aspects of the stability analysis of nonlinear structures. Comput. Methods Appl. Mech. Eng. 47: 219-259

Rybicki E F, Kanninen M F 1977 A finite element calculation of stress intensity factors by a modified crack closure integral. Eng. Fracture Mech. 9: 931-938

Tsao D, Duan D, Ji W 1991 Delamination fracture analysis of composite laminates caused by local buckling and postbuckling. SAE Trans. 100 (sect. 1; pt 2;911986): 2034-2043

Wagner W, Wriggers 1988 A simple method for the calculation of post-critical branches. Eng. Comput. 5: 103-109

Wempner G A 1979 Discrete approximations related to nonlinear theories of solids. Int. J. Solids Struct. 7: 1581-1599

Whitcomb J D 19893-dimensional analysis of a post-buckled embedded delamination. J. Compos. Mater. 23: 862-889

Whitcomb J D, Shivakumar K N 1989 Strain energy release rate analysis of plates with postbuckled delaminations. J. Compos. Mater. 23: 714-734

Yin W L 1984 The energy release rate in the growth of a 1-dimensional delamination. J. Appl. Mech. 51: 939-941

Yin W L 1985 Axisymmetric buckling and growth of a circular delamination in a compressed laminate. Int. J. Solids and Struct. 21: 503-514 